\title{
Cramer-Rao bounds of DOA estimates for BPSK and QPSK modulated signals
}

\author{
Jean Pierre Delmas, Member IEEE, Habti Abeida \\ GET/INT, Département CITI, UMR-CNRS 5157, \\ 9 rue Charles Fourier, 91011 Evry Cedex, FRANCE. \\ LSTA, Université Paris VI. \\ Fax: +33-1-60 7644 33, e-mail: jean-pierre.delmas(habti.abeida)@int-evry.fr.
}

\begin{abstract}
This paper focuses on the stochastic CramerRao bound (CRB) of direction of arrival (DOA) estimates for binary phase-shift keying (BPSK) and quaternary phase-shift keying (QPSK) modulated signals corrupted by additive circular complex Gaussian noise. Explicit expressions of the CRB for the DOA parameter alone in the case of a single signal waveform are given. These CRB's are compared, on the one hand, with those obtained with different a priori knowledge, and on the other hand with CRB's under the non-circular and circular complex Gaussian distribution and with different deterministic CRB's. It is shown in particular, that the CRB's under the non-circular [resp. circular] complex Gaussian distribution are tight upper bounds on the CRB's under the BPSK [resp. QPSK] distribution at very low and very high signal to noise ratios (SNR) only. Finally, these results and comparisons are extended to the case of two independent BPSK or QPSK distributed sources where an explicit expression of the CRB for the DOA parameters alone is given for large SNR.
\end{abstract}

Keywords - Stochastic Cramer-Rao bound, DOA estimation, Fisher information matrix, BPSK and QPSK signals.

\section{INTRODUCTION}

$\mathrm{D}$ ETERMINISTIC and stochastic CRB's play an important role in DOA estimation because they serve as a benchmark for the performance of actual estimators (see e.g., [1]). Moreover, the stochastic CRB can be achieved asymptotically (in the number of measurements) by the stochastic maximum likelihood (ML) method. Unfortunately, this stochastic CRB appears to be prohibitive to compute for non-Gaussian processes including discrete signal waveforms. And to the best of our knowledge, no contribution has dealt with stochastic CRB for discrete signal waveforms in DOA estimation yet, despite some recent works on stochastic CRB for non-circular signals (e.g., [2],[3]). To cope with this difficulty, a method sometimes used is to assume that the signals are arbitrary deterministic sequences while the noise is circular complex Gaussian, so that the distribution is still Gaussian and the associated deterministic CRB is easily deduced (see e.g., [4, rel. (2.13)]). But the corresponding deterministic (or conditional) ML method does not achieve this deterministic CRB because the deterministic likelihood function does not meet the required regularity conditions. Consequently, this deterministic CRB is only a non-attainable lower bound on the variance of any unbiased DOA estimator. To deal with non-Gaussian processes, another solution is to suppose that the signals are Gaussian but not necessarily complex circular. In that case, the associated CRB is under rather general conditions (see e.g., [5, p. 293]) the largest CRB among the class of arbitrary distributions with given covariance matrices. This approach was used in [6] for non-circular complex signal waveforms such as discrete signals. But the associated CRB is only an upper bound on the true stochastic CRB. Faced with the drawbacks of the two aforementioned approximations, we need an explicit expression of the stochastic CRB under non-Gaussian distributions.

In this paper, we derive explicit expressions of the stochastic CRB for the DOA parameter alone in the case of BPSK and QPSK signal waveforms observed in additive circular complex Gaussian noise. More specifically, the main contribution of this paper is devoted to the case of a single BPSK and QPSK signal waveform. Because the distribution of these models are simple mixed Gaussian, an explicit expression of the Fisher information matrix (FIM) is derived using well known properties of the Gaussian distribution. An explicit expression of the stochastic CRB for the DOA parameter alone is deduced. We note that apart from the DOA applications, the recent papers (e.g., [7], [8]) that deal with stochastic CRB's for estimating the carrier phase and frequency of BPSK and QPSK waveforms, do not give analytic solutions (see e.g., [7, rels.(9)(16)], [8, rels.(16)]). Our CRB's are compared with those obtained with different a priori knowledge and are confronted with the non-circular and circular complex Gaussian CRB's and with different deterministic CRB's presented in [9]. It is shown in particular, that the CRB's under the non-circular [resp. circular] complex Gaussian distribution are tight upper bounds on the CRB's under the BPSK [resp. QPSK] distribution at very low and very high SNR's only. Finally, the case of two independent BPSK distributed sources is dealt with. Due to the computational complexity, an explicit expression of the DOA parameters alone is given for large SNR only. Furthermore numerical comparisons of the stochastic CRB's associated with BPSK and noncircular Gaussian distributed sources are given for all SNR's. In particular, they show that the CRB under the 
non-circular Gaussian distribution is a very loose upper bound on the CRB under the BPSK distribution. And the difference between these CRB's is more prominent for small DOA and phase separation.

The following notations are used throughout the paper. Matrices and vectors are represented by bold upper case and bold lower case characters, respectively. Vectors are by default in column orientation, while $T, H, *$, $\Re, \Im$ and $i$ stand for transpose, conjugate transpose, conjugate, real and imaginary part and $\sqrt{-1}$, respectively. $\otimes$ is the standard Kronecker product of matrices. The symbol $1_{A}$ denotes the indicator function of the condition $A$, which assumes the value 1 if this condition is satisfied and 0 otherwise, and the symbol $\mathcal{N}(m ; v)$ denotes the univariate Gaussian distribution with mean $m$ and variance $v$.

\section{DATA MODEL}

Consider a BPSK or QPSK modulated signal impinging on an arbitrary array of $M$ sensors. We assume that the array is perfectly calibrated for which the steering vector is a kwown function of the source's DOA. The received signals are bandpass filtered and after downshifting the sensor signal to baseband, the in-phase and quadrature components are paired to obtain complex signals. We assume Nyquist shaping and ideal sample timing so that the inter-symbol interference at each symbol spaced sampling instance can be ignored. In the absence of frequency offset but with possible phase offset, the signals at the output of the matched filter can be represented as:

$$
\mathbf{y}_{t}=s_{t} \mathbf{a}_{1}+\mathbf{n}_{t} \quad t=1, \ldots, T
$$

where $\mathbf{a}_{1}$ is the steering vector parametrized by the scalar DOA parameter $\theta_{1}$. We suppose $\left\|\mathbf{a}_{1}\right\|^{2}=M$. $s_{t}=\sigma_{1} e^{i \phi_{1}} \epsilon_{t}$ where $\left(\epsilon_{t}\right)_{t=1, \ldots, T}$ are independent, identically, distributed (IID) random symbols taking values \pm 1 [resp. $\pm \sqrt{2} / 2 \pm i \sqrt{2} / 2$ ] with equal probabilities for BPSK [resp. QPSK] modulations, where $\phi_{1}$ and $\sigma_{1}$ are considered as unknown parameters. The symbols $\epsilon_{t}$ are assumed to be independent from $\mathbf{n}_{t}$. $\left(\mathbf{n}_{t}\right)_{t=1, \ldots, T}$ are IID $M$-variate zero-mean complex circular Gaussian random vectors with $\mathrm{E}\left(\mathbf{n}_{t} \mathbf{n}_{t}^{H}\right)=\sigma_{n}^{2} \mathbf{I}_{M}$. Consequently $\left(\mathbf{y}_{t}\right)_{t=1, \ldots, T}$ are IID $M$-dimensional random variable whose probability density function (PDF) is mixed circular Gaussian:

$$
p\left(\mathbf{y}_{t} ; \boldsymbol{\alpha}\right)=\frac{1}{L \pi^{M} \sigma_{n}^{2 M}} \sum_{l=1}^{L} e^{-\frac{\| \mathbf{y}_{t}-\sigma_{1} e^{i \phi_{1} \epsilon_{l} \mathbf{a}_{1} \|^{2}}}{\sigma_{n}^{2}}}
$$

with $L=2$ and $\epsilon_{l}= \pm 1$ [resp. $L=4$ and $\epsilon_{l}= \pm \sqrt{2} / 2 \pm$ $i \sqrt{2} / 2$ ] for BPSK [resp. QPSK] modulated signals and where $\boldsymbol{\alpha} \stackrel{\text { def }}{=}\left(\sigma_{n}, \sigma_{1}, \phi_{1}, \theta_{1}\right)^{T}$.

\section{Stochastic CRB FOR BPSK AND QPSK SIGNALS}

The main result of the paper proved in Appendix A is contained in the following theorem
Theorem 1: The FIM associated with the parameter $\left(\sigma_{n}, \sigma_{1}, \phi_{1}, \theta_{1}\right)$ of stochastic BPSK and QPSK modulated signals are given by the explicit closed-form expressions:

$$
\begin{aligned}
& \mathbf{I}_{\mathrm{F}}^{\mathrm{BPSK}}=T\left[\begin{array}{cc}
\mathbf{I}_{\mathrm{F}_{1}}^{\mathrm{BPK}} & \mathbf{O} \\
\mathbf{O} & \mathbf{I}_{\mathrm{F}_{2}}^{\mathrm{BPSK}}
\end{array}\right], \\
& \mathbf{I}_{\mathrm{F}}^{\mathrm{QPSK}}=T\left[\begin{array}{cc}
\mathbf{I}_{\mathrm{F}_{1}}^{\mathrm{QPSK}} & \mathbf{O} \\
\mathbf{O} & \mathbf{I}_{\mathrm{F}_{2}}^{\mathrm{QPSK}}
\end{array}\right]
\end{aligned}
$$

with

$$
\mathbf{I}_{\mathrm{F}_{1}}^{\mathrm{BPSK}}=\left[\begin{array}{cc}
\frac{4 M}{\sigma_{n}^{2}}\left(1-\frac{2 \sigma_{1}^{2}}{\sigma_{n}^{2}} f_{1}(\rho)\right) & \frac{4 M \sigma_{1}}{\sigma_{n}^{3}} f_{1}(\rho) \\
\frac{4 M \sigma_{1}}{\sigma_{n}^{3}} f_{1}(\rho) & \frac{2 M}{\sigma_{n}^{2}}\left(1-f_{1}(\rho)\right)
\end{array}\right]
$$

$$
\begin{gathered}
\mathbf{I}_{\mathrm{F}_{2}}^{\mathrm{BPSK}}=\left[\begin{array}{c}
\frac{2 M \sigma_{1}^{2}}{\sigma_{n}^{2}}\left(1-f_{2}(\rho)\right) \\
\frac{2 \sigma_{1}^{2}\left(i \mathbf{a}_{1}^{\prime} \mathbf{a}_{1}\right)}{\sigma_{n}^{2}}\left(1-f_{2}(\rho)\right) \\
\frac{2 \sigma_{1}^{2}\left(i \mathbf{a}_{1}^{\prime} \mathbf{a}_{1}\right)}{\sigma_{n}^{2}}\left(1-f_{2}(\rho)\right) \\
\frac{2 \sigma_{1}^{2}\left\|\mathbf{a}_{1}^{\prime}\right\|^{2}}{\sigma_{n}^{2}}\left(1-f_{2}(\rho)\right)
\end{array}\right] \\
\mathbf{I}_{\mathrm{F}_{1}}^{\mathrm{QPSK}}=\left[\begin{array}{cc}
\frac{4 M}{\sigma_{n}^{2}}\left(1-\frac{2 \sigma_{1}^{2}}{\sigma_{n}^{2}} f_{1}\left(\frac{\rho}{2}\right)\right) & \frac{4 M \sigma_{1}}{\sigma_{n}^{3}} f_{1}\left(\frac{\rho}{2}\right) \\
\frac{4 M \sigma_{1}}{\sigma_{n}^{3}} f_{1}\left(\frac{\rho}{2}\right) & \frac{2 M}{\sigma_{n}^{2}}\left(1-f_{1}\left(\frac{\rho}{2}\right)\right)
\end{array}\right] \\
\mathbf{I}_{\mathrm{F}_{2}}^{\mathrm{QPSK}}=\left[\begin{array}{cc}
\frac{2 M \sigma_{1}^{2}}{\sigma_{n}^{2}}\left(1-(1+\rho) f_{2}\left(\frac{\rho}{2}\right)\right) \\
\frac{2 \sigma_{1}^{2}\left(i \mathbf{a}_{1}^{\prime} \mathbf{a}_{1}\right)}{\sigma_{n}^{2}}\left(1-(1+\rho) f_{2}\left(\frac{\rho}{2}\right)\right) \\
\frac{2 \sigma_{1}^{2}\left(i \mathbf{a}_{1}^{\prime} \mathbf{a}_{1}\right)}{\sigma_{n}^{2}}\left(1-(1+\rho) f_{2}\left(\frac{\rho}{2}\right)\right) \\
\frac{2 \sigma_{1}^{2}\left\|\mathbf{a}_{1}^{\prime}\right\|^{2}}{\sigma_{n}^{2}}\left(1-\left(1+\frac{\rho}{M} \frac{\left|\mathbf{a}_{1}^{H} \mathbf{a}_{1}^{\prime}\right|^{2}}{\left\|\mathbf{a}_{1}^{\prime}\right\|^{2}}\right) f_{2}\left(\frac{\rho}{2}\right)\right)
\end{array}\right]
\end{gathered}
$$

with $\rho \stackrel{\text { def }}{=} \frac{M \sigma_{1}^{2}}{\sigma_{n}^{2}}$ and $\mathbf{a}_{1}^{\prime} \stackrel{\text { def }}{=} \frac{d \mathbf{a}_{1}}{d \theta_{1}}$ and where $f_{1}$ and $f_{2}$ are the following decreasing function of $\rho: f_{1}(\rho) \stackrel{\text { def }}{=}$ $\frac{e^{-\rho}}{\sqrt{2 \pi}} \int_{-\infty}^{+\infty} \frac{u^{2} e^{-\frac{u^{2}}{2}}}{\cosh (u \sqrt{2 \rho})} d u, f_{2}(\rho) \stackrel{\text { def }}{=} \frac{e^{-\rho}}{\sqrt{2 \pi}} \int_{-\infty}^{+\infty} \frac{e^{-\frac{u^{2}}{2}}}{\cosh (u \sqrt{2 \rho})} d u$ We note the similarity of the $2 \times 2$ top left corner of these FIM's with those derived in [10] used for the estimation of the SNR of BPSK and QPSK modulated signals. Because these Fisher information matrices are block diagonal, the following explicit expressions for the CRB for the parameter DOA alone are easily derived:

$$
\begin{aligned}
\operatorname{CRB}_{\text {BPSK }}\left(\theta_{1}\right) & =\frac{1}{T}\left(\frac{1}{\gamma_{1}} \frac{\sigma_{n}^{2}}{\sigma_{1}^{2}}\right)\left(\frac{1}{1-f_{2}(\rho)}\right) \\
\operatorname{CRB}_{\mathrm{QPSK}}\left(\theta_{1}\right) & =\frac{1}{T}\left(\frac{1}{\gamma_{1}} \frac{\sigma_{n}^{2}}{\sigma_{1}^{2}}\right)\left(\frac{1}{1-f_{2}\left(\frac{\rho}{2}\right)}\right)
\end{aligned}
$$

where $\gamma_{1}$ is the purely geometrical factor $2 \mathbf{a}_{1}^{\prime H} \boldsymbol{\Pi}_{\mathbf{a}_{1}}^{\perp} \mathbf{a}_{1}^{\prime}$ with $\boldsymbol{\Pi}_{\mathbf{a}_{1}}^{\perp} \stackrel{\text { def }}{=} \mathbf{I}_{M}-\frac{\mathbf{a}_{1} \mathbf{a}_{1}^{H}}{M}$. We note that thanks to the decreasing function $f_{2}, \operatorname{CRB}_{\mathrm{BPSK}}\left(\theta_{1}\right)<\operatorname{CRB}_{\mathrm{QPSK}}\left(\theta_{1}\right)$. 
In the absence of phase offset or after correcting it (i.e., parameter $\phi_{1}$ known), these CRB's for $\theta_{1}$ become

$$
\begin{aligned}
\operatorname{CRB}_{\mathrm{BPSK}}^{\mathrm{CO}}\left(\theta_{1}\right)= & \frac{1}{T}\left(\frac{1}{2\left\|\mathbf{a}_{1}^{\prime}\right\|^{2}} \frac{\sigma_{n}^{2}}{\sigma_{1}^{2}}\right)\left(\frac{1}{1-f_{2}(\rho)}\right)(3) \\
\operatorname{CRB}_{\mathrm{QPSK}}^{\mathrm{CO}}\left(\theta_{1}\right)= & \frac{1}{T}\left(\frac{1}{2\left\|\mathbf{a}_{1}^{\prime}\right\|^{2}} \frac{\sigma_{n}^{2}}{\sigma_{1}^{2}}\right) \\
& \left(\frac{1}{1-\left(1+\frac{\rho}{M} \frac{\left|\mathbf{a}_{1}^{H} \mathbf{a}_{1}^{\prime}\right|^{2}}{\left\|\mathbf{a}_{1}^{\prime}\right\|^{2}}\right) f_{2}\left(\frac{\rho}{2}\right)}\right)(4)
\end{aligned}
$$

Comparing (3) to (1), we note that the phase information for a BPSK source is quite informative for all SNR's because $2\left\|\mathbf{a}_{1}^{\prime}\right\|^{2}>\gamma_{1}$ for the DOA perspective contrary to a QPSK source, as shown in section V-A (Fig.3).

\section{COMParison With RElated CRB's}

Depending on the presence of different a priori information on the parameters and on the distribution of the sources, several CRB's can be considered.

Data-aided CRB: If we assume that transmitted symbols are known at the array receiver, the modulation can be removed perfectly and the resulting signal $\mathbf{y}_{t}$ is circular Gaussian distributed with mean $\sigma_{1} e^{i \phi_{1}} \epsilon_{t} \mathbf{a}_{1}$ and covariance $\sigma_{n}^{2} \mathbf{I}_{M}$ and consequently $\left(\mathbf{y}_{1}^{T}, \ldots, \mathbf{y}_{T}^{T}\right)^{T}$ is circular Gaussian distributed as well, with mean $\boldsymbol{\epsilon} \otimes \sigma_{1} e^{i \phi_{1}} \mathbf{a}_{1}$ and covariance $\sigma_{n}^{2} \mathbf{I}_{M T}$ where $\boldsymbol{\epsilon} \stackrel{\text { def }}{=}\left(\epsilon_{1}, \ldots, \epsilon_{T}\right)^{T}$. Applying the Slepian-Bangs formula (see e.g., [5, rel. (B.3.25)]), we obtain

$$
\begin{aligned}
\left(\mathbf{I}_{\mathrm{F}}^{\mathrm{DA}}\right)_{k, l} & =\frac{1}{\sigma_{n}^{4}} \operatorname{Tr}\left(\frac{\partial \sigma_{n}^{2} \mathbf{I}_{M T}}{\partial \alpha_{k}} \frac{\partial \sigma_{n}^{2} \mathbf{I}_{M T}}{\partial \alpha_{l}}\right) \\
& +\frac{2}{\sigma_{n}^{2}} \sum_{t=1}^{T}\left|\epsilon_{t}\right|^{2} \Re\left(\frac{\partial \sigma_{1} e^{-i \phi_{1}} \mathbf{a}_{1}^{H}}{\partial \alpha_{k}} \frac{\partial \sigma_{1} e^{i \phi_{1}} \mathbf{a}_{1}}{\partial \alpha_{l}}\right),
\end{aligned}
$$

then the following FIM is straightforwardly derived by noting that $\frac{1}{T} \sum_{t=1}^{T}\left|\epsilon_{t}\right|^{2}=1$ and $\left\|\mathbf{a}_{1}\right\|^{2}=M$ which implies $\mathbf{a}_{1}^{H} \mathbf{a}_{1}^{\prime}+\left(\mathbf{a}_{1}^{H} \mathbf{a}_{1}^{\prime}\right)^{H}=0$, and consequently $\Re\left(\mathbf{a}_{1}^{H} \mathbf{a}_{1}^{\prime}\right)=$ 0 and $\Im\left(\mathbf{a}_{1}^{H} \mathbf{a}_{1}^{\prime}\right)=i \mathbf{a}_{1}^{\prime}{ }^{H} \mathbf{a}_{1}$ :

$$
\mathbf{I}_{\mathrm{F}}^{\mathrm{DA}}=T\left[\begin{array}{cccc}
\frac{4 M}{\sigma_{n}^{2}} & 0 & 0 & 0 \\
0 & \frac{2 M}{\sigma_{n}^{2}} & 0 & 0 \\
0 & 0 & \frac{2 M \sigma_{1}^{2}}{\sigma_{n}^{2}} & \frac{2 \sigma_{1}^{2}\left(i \mathbf{a}_{1}^{\prime}{ }^{H} \mathbf{a}_{1}\right)}{\sigma_{n}^{2}} \\
0 & 0 & \frac{2 \sigma_{1}^{2}\left(i \mathbf{a}_{1}^{\prime} \mathbf{a}_{1}\right)}{\sigma_{n}^{2}} & \frac{2 \sigma_{1}^{2}\left\|\mathbf{a}_{1}\right\|^{2}}{\sigma_{n}^{2}}
\end{array}\right]
$$

and consequently

$$
\mathrm{CRB}_{\mathrm{DA}}\left(\theta_{1}\right)=\frac{1}{T}\left(\frac{1}{\gamma_{1}} \frac{\sigma_{n}^{2}}{\sigma_{1}^{2}}\right) .
$$

Because $\lim _{\rho \rightarrow \infty} f_{1}(\rho)=\lim _{\rho \rightarrow \infty} f_{2}(\rho)=0$, we note that the FIM's $\mathbf{I}_{\mathrm{F}}^{\mathrm{BPSK}}$ and $\mathbf{I}_{\mathrm{F}}^{\mathrm{QPSK}}$ of section III approach

\footnotetext{
${ }^{1}$ Where the exponent $\mathrm{CO}$ of $\mathrm{CRB}_{\mathrm{BPSK}}^{\mathrm{CO}}\left(\theta_{1}\right)$ and $\mathrm{CRB}_{\mathrm{QPSK}}^{\mathrm{CO}}\left(\theta_{1}\right)$ means coherent.
}

$\mathbf{I}_{\mathrm{F}}^{\mathrm{DA}}$ and consequently $\operatorname{CRB}_{\mathrm{BPSK}}\left(\theta_{1}\right)$ and $\operatorname{CRB}_{\mathrm{QPSK}}\left(\theta_{1}\right)$ approach $\operatorname{CRB}^{\mathrm{DA}}\left(\theta_{1}\right)$ for large $\mathrm{SNR}$ values. On the contrary, because $2\left\|\mathbf{a}_{1}^{\prime}\right\|^{2}>\gamma_{1}, \operatorname{CRB}_{\mathrm{BPSK}}^{\mathrm{CO}}\left(\theta_{1}\right)$ and $\operatorname{CRB}_{\mathrm{QPSK}}^{\mathrm{CO}}\left(\theta_{1}\right)$ are lower than $\operatorname{CRB}^{\mathrm{DA}}\left(\theta_{1}\right)$ for large SNR values. The phase information is quite informative compared with the training symbols from the DOA perspective.

Deterministic CRB: For the deterministic or conditional model of the signal waveform, the CRB for $\theta_{1}$ does not depend on the realization of $\left(\epsilon_{t}\right)_{t=1, \ldots, T}$ because $\frac{1}{T} \sum_{t=1}^{T}\left|\epsilon_{t}\right|^{2}=1$ and is given by (see e.g., [4, rel. $(2.11)])$

$$
\operatorname{CRB}_{\text {DET }}\left(\theta_{1}\right)=\frac{1}{T}\left(\frac{1}{\gamma_{1}} \frac{\sigma_{n}^{2}}{\sigma_{1}^{2}}\right) .
$$

We note that if side information is available such as the constant modulus of BPSK and QPSK modulation [11] and some training symbols among the $T$ symbols $\epsilon_{t}[9$, rel. (50)], the previous CRB for $\theta_{1}$ is preserved. Furthermore, the data-aided and deterministic CRB are the same, implying that knowing the signal or not is not important.

Stochastic complex Gaussian CRB: Because the BPSK [resp. QPSK] modulation is non-circular [resp. circular] complex to the second-order, it makes sense to compare the stochastic CRB's (1) and (2) to the CRB's associated with respectively non-circular ${ }^{2}$ (NCG) [6, rel. (3.14)] or circular (CG) complex Gaussian distribution that can be considered as upper bounds on the true stochastic CRB's (see e.g., [5, p. 293]). More precisely, after recalling these CRB's under Gaussian distributions for the convenience of the reader

$$
\begin{aligned}
\operatorname{CRB}_{\mathrm{NCG}}\left(\theta_{1}\right) & =\frac{1}{T}\left(\frac{1}{\gamma_{1}}\left[\frac{\sigma_{n}^{2}}{\sigma_{1}^{2}}+\frac{1}{2 M} \frac{\sigma_{n}^{4}}{\sigma_{1}^{4}}\right]\right), \\
\operatorname{CRB}_{\mathrm{CG}}\left(\theta_{1}\right) & =\frac{1}{T}\left(\frac{1}{\gamma_{1}}\left[\frac{\sigma_{n}^{2}}{\sigma_{1}^{2}}+\frac{1}{M} \frac{\sigma_{n}^{4}}{\sigma_{1}^{4}}\right]\right),
\end{aligned}
$$

we have

$$
\frac{\operatorname{CRB}_{\mathrm{BPSK}}\left(\theta_{1}\right)}{\operatorname{CRB}_{\mathrm{NCG}}\left(\theta_{1}\right)}=\frac{1}{\left(1-f_{2}(\rho)\right)\left(1+\frac{1}{2 \rho}\right)}
$$

and

$$
\frac{\operatorname{CRB}_{\mathrm{QPSK}}\left(\theta_{1}\right)}{\mathrm{CRB}_{\mathrm{CG}}\left(\theta_{1}\right)}=\frac{1}{\left(1-f_{2}\left(\frac{\rho}{2}\right)\right)\left(1+\frac{1}{\rho}\right)}
$$

We note that these ratios depend on $\rho \stackrel{\text { def }}{=} \frac{M \sigma_{1}^{2}}{\sigma_{n}^{2}}$ only and tend to 1 when $\rho$ tends to $\infty$. However this dependence in $\rho$ is not monotone as it is numerically shown in the next section.

\footnotetext{
${ }^{2}$ Because $\mathrm{E}\left(\epsilon_{t}^{2}\right)=\mathrm{E}\left|\epsilon_{t}^{2}\right|$ for the BPSK modulation, we consider the non-circular complex Gaussian distribution associated with $\mathrm{E}\left(\epsilon_{t}^{2}\right)=\mathrm{E}\left|\epsilon_{t}^{2}\right|=1$, i.e., with $\rho_{1}=1$ in $[6$, rel. (3.14)] where the non-circularity rate $\rho_{1}$ and the circularity phase $\phi_{1}$ of $\epsilon_{t}$ are defined here by $\mathrm{E}\left(\epsilon_{t}^{2}\right)=\rho_{1} e^{2 i \phi_{1}} \mathrm{E}\left|\epsilon_{t}^{2}\right|$.
} 


\section{NuMERICAL EXAMPLES}

The purpose of this section is to illustrate the results of section IV and to extend them to the case of two independent BPSK distributed sources. We consider throughout this section, one or two independent sources impinging on a uniform linear array (ULA) of $M$ sensors spaced a half-wavelength apart for which $\mathbf{a}_{k}=\left(1, e^{i \theta_{k}}, \ldots, e^{i(M-1) \theta_{k}}\right)^{T}$.

\section{A. Single source case}

The first experiment illustrates the results of section IV. Fig. 1 shows the ratios $\frac{\operatorname{CRB}_{\mathrm{BPSK}}\left(\theta_{1}\right)}{\operatorname{CRB}_{\mathrm{NCG}}\left(\theta_{1}\right)}$ and $\frac{\operatorname{CRB}_{\mathrm{QPSK}}\left(\theta_{1}\right)}{\operatorname{CRB}_{\mathrm{CG}}\left(\theta_{1}\right)}$ as a function of $\rho \stackrel{\text { def }}{=} \frac{M \sigma_{1}^{2}}{\sigma_{n}^{2}}$. We see from that figure that the CRB's under the non-circular [resp. circular] complex Gaussian distribution are tight upper bounds on the CRB's under the BPSK [resp. QPSK] distribution at very low and very high SNR's only.

Figs. 2 and 3 show the different CRB's ${ }^{3}$ as a function of the SNR for the BPSK and QPSK modulations respectively. From these figures, we see that the CRB's achieved in the absence of phase offset outperform all the other CRB's except for very low SNR's. It is shown that there is no significant difference between the bounds for non-dataaided and data-aided estimations except at low SNR.

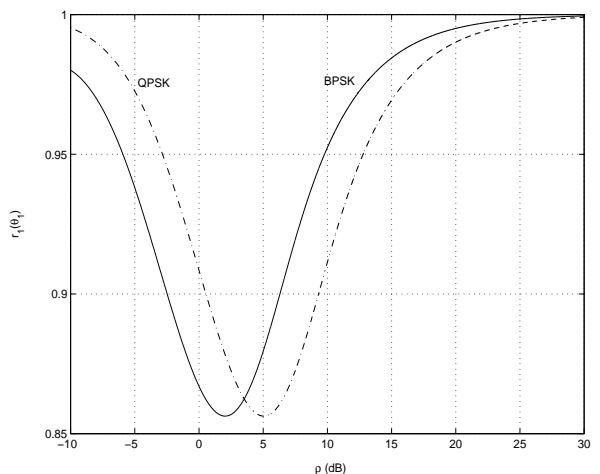

Fig.1 Ratios $r_{1}\left(\theta_{1}\right) \stackrel{\text { def }}{=} \frac{\operatorname{CRB}_{\mathrm{BPSK}}\left(\theta_{1}\right)}{\operatorname{CRB}_{\mathrm{NCG}}\left(\theta_{1}\right)}$ and $r_{1}\left(\theta_{1}\right) \stackrel{\text { def }}{=} \frac{\operatorname{CRB}_{\mathrm{QPSK}}\left(\theta_{1}\right)}{\operatorname{CRB}_{\mathrm{CG}}\left(\theta_{1}\right)}$ as a function of $\rho \stackrel{\text { def }}{=} \frac{M \sigma_{1}^{2}}{\sigma_{n}^{2}}$.

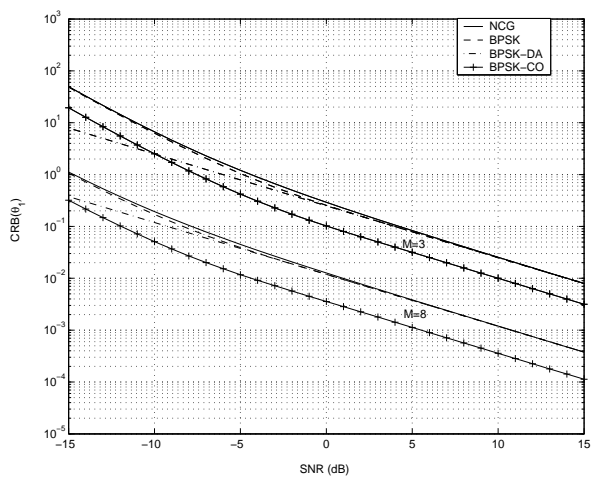

\footnotetext{
${ }^{3}$ All the CRB's are computed for $T=1$. That means that the actual CRB's associated with the signal model defined in section II are obtained from the results given in this section by dividing by $T$.
}

Fig.2 Normalized $(T=1)$ CRB's for BPSK modulation: $\operatorname{CRB}_{\mathrm{NCG}}\left(\theta_{1}\right), \operatorname{CRB}_{\mathrm{BPSK}}\left(\theta_{1}\right), \mathrm{CRB}_{\mathrm{BPSK}}^{\mathrm{DA}}\left(\theta_{1}\right)$ and $\operatorname{CRB}_{\mathrm{BPSK}}^{\mathrm{CO}}\left(\theta_{1}\right)$ as a function of the SNR.

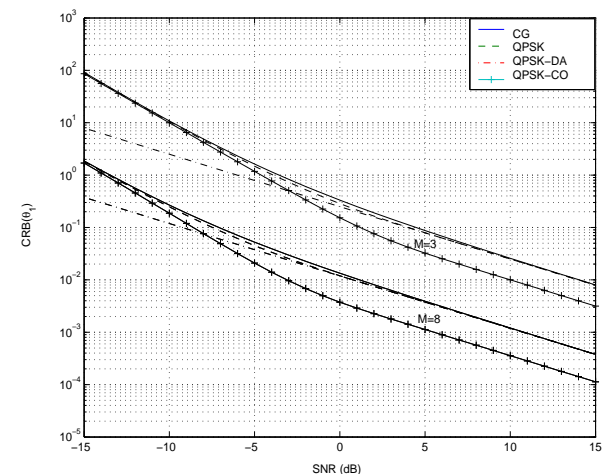

Fig.3 Normalized $(T=1)$ CRB's for QPSK modulation: $\operatorname{CRB}_{\mathrm{CG}}\left(\theta_{1}\right), \operatorname{CRB}_{\mathrm{QPSK}}\left(\theta_{1}\right), \operatorname{CRB}_{\mathrm{QPSK}}^{\mathrm{DA}}\left(\theta_{1}\right)$ and $\operatorname{CRB}_{\mathrm{QPSK}}^{\mathrm{CO}}\left(\theta_{1}\right)$ as a function of the SNR.

\section{B. Two sources case}

We consider now two independent BPSK or QPSK distributed sources. Because the PDF of $\mathbf{y}_{t}$ is a mixture of 4 or 16 Gaussian PDFs, the associated stochastic CRB appears to be prohibitive to compute. Consequently we use a numerical approximation derived from the strong law of large numbers, i.e.

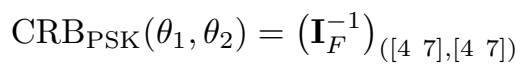

with

$$
\frac{1}{T}\left(\mathbf{I}_{F}\right)_{k, l}=\lim _{T^{\prime} \rightarrow \infty} \frac{1}{T^{\prime}} \sum_{t=1}^{T^{\prime}}\left(\frac{\partial \ln p\left(\mathbf{y}_{t} ; \boldsymbol{\alpha}\right)}{\partial \alpha_{k}}\right)\left(\frac{\partial \ln p\left(\mathbf{y}_{t} ; \boldsymbol{\alpha}\right)}{\partial \alpha_{l}}\right)
$$

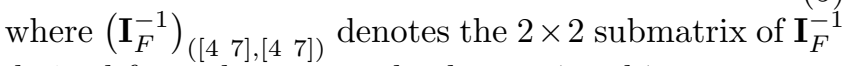
derived from the rows and columns 4 and 7 .

$$
p\left(\mathbf{y}_{t} ; \boldsymbol{\alpha}\right)=\frac{1}{L^{2} \pi^{M} \sigma_{n}^{2 M}} \sum_{j=1}^{L^{2}} e^{-\frac{\left\|\mathbf{y}_{t}-\mathbf{A s}_{j}\right\|^{2}}{\sigma_{n}^{2}}}
$$

with $\mathbf{s}_{j} \stackrel{\text { def }}{=}\left(\sigma_{1} \eta_{j, 1} e^{i \phi_{1}}, \sigma_{2} \eta_{j, 2} e^{i \phi_{2}}\right)^{T}$ with $L=2$ [resp. $L=4]$ for BPSK [resp. QPSK] modulated signals where $\left(\eta_{j, 1}, \eta_{j, 2}\right)_{j=1 \ldots, L^{2}}=( \pm 1, \pm 1)$ [resp. $( \pm \sqrt{2} / 2 \pm i \sqrt{2} / 2, \pm \sqrt{2} / 2 \pm i \sqrt{2} / 2)]$ and where $\boldsymbol{\alpha} \stackrel{\text { def }}{=}$ $\left(\sigma_{n}, \sigma_{1}, \phi_{1}, \theta_{1}, \sigma_{2}, \phi_{2}, \theta_{2}\right)^{T}$ and $\mathbf{A} \stackrel{\text { def }}{=}\left(\mathbf{a}_{1}, \mathbf{a}_{2}\right)$. At high SNR's (more precisely for $M \frac{\sigma_{1}^{2}}{\sigma_{n}^{2}} \gg 1$ and $M \frac{\sigma_{2}^{2}}{\sigma_{n}^{2}} \gg 1$ ) it is proved in Appendix B that the FIM associated with BPSK and QPSK signals are approximated by the same following explicit expression:

$$
\mathbf{I}_{\mathrm{F}}^{\mathrm{BPSK}} \approx \mathbf{I}_{\mathrm{F}}^{\mathrm{QPSK}} \approx T\left[\begin{array}{ccc}
\frac{4 M}{\sigma_{n}^{2}} & \mathbf{0}^{T} & \mathbf{0}^{T} \\
\mathbf{0} & \mathbf{I}_{1} & \mathbf{O} \\
\mathbf{0} & \mathbf{O} & \mathbf{I}_{2}
\end{array}\right]
$$


with $\mathbf{I}_{k}=\left[\begin{array}{ccc}\frac{2 M}{\sigma_{n}^{2}} & 0 & 0 \\ 0 & \frac{2 M \sigma_{k}^{2}}{\sigma_{n}^{2}} & \frac{2 \sigma_{k}^{2}\left(i \mathbf{a}_{k}^{\prime}{ }^{\prime} \mathbf{a}_{k}\right)}{\sigma_{n}^{2}} \\ 0 & \frac{2 \sigma_{k}^{2}\left(i \mathbf{a}_{k}^{\prime} \mathbf{a}_{k}\right)}{\sigma_{n}^{2}} & \frac{2 \sigma_{k}^{2}\left\|\mathbf{a}_{k}^{\prime}\right\|^{2}}{\sigma_{n}^{2}}\end{array}\right], k=1,2$.

We clearly see that the entries corresponding to sources 1 and 2 are decoupled. Consequently, for large SNR's and independent sources, the CRB for the DOA of one source is independent of the parameters of the other source and

$$
\begin{aligned}
\operatorname{CRB}_{\mathrm{BPSK}}\left(\theta_{1}, \theta_{2}\right) & \approx \operatorname{CRB}_{\mathrm{QPSK}}\left(\theta_{1}, \theta_{2}\right) \\
& \approx \frac{1}{T}\left[\begin{array}{cc}
\frac{1}{\gamma_{1}} \frac{\sigma_{n}^{2}}{\sigma_{1}^{2}} & 0 \\
0 & \frac{1}{\gamma_{2}} \frac{\sigma_{n}^{2}}{\sigma_{2}^{2}}
\end{array}\right]
\end{aligned}
$$

where $\gamma_{i} \stackrel{\text { def }}{=} 2 \mathbf{a}_{i}^{\prime H} \boldsymbol{\Pi}_{\mathbf{a}_{i}}^{\perp} \mathbf{a}_{i}^{\prime}, i=1,2$. This quite curious result reminds one of a similar result in cissoid parameter estimation (see e.g., [12]) where the asymptotic $\mathrm{CRB}$ of the frequencies is independent of the frequency separation. Furthermore, the CRB's $\operatorname{CRB}_{\mathrm{BPSK}}\left(\theta_{k}\right) \approx$ $\operatorname{CRB}_{\mathrm{QPSK}}\left(\theta_{k}\right)$ and $\operatorname{CRB}_{\mathrm{BPSK}}^{\mathrm{CO}}\left(\theta_{k}\right) \approx \operatorname{CRB}_{\mathrm{QPSK}}^{\mathrm{CO}}\left(\theta_{k}\right)$ for each DOA are those of the single source case (because the $4 \times 4$ top left corner of the FIM (6) is the FIM given in theorem 1 for high SNR). We note that this property is quite different from the behavior of the CRB under the Gaussian distribution and the deterministic CRB, for which the CRB for the DOA of one source depends on the DOA separation. More precisely, it is proved [4, result R9] that these latter two CRB's tend to the same limit as all SNR's increase. For independent sources, they are given by (from e.g. [4, rel. (2.13)])

$$
\begin{aligned}
\operatorname{CRB}_{\mathrm{DET}}\left(\theta_{1}, \theta_{2}\right) & =\mathrm{CRB}_{\mathrm{CG}}\left(\theta_{1}, \theta_{2}\right) \\
& =\frac{1}{T}\left[\begin{array}{cc}
\frac{1}{\beta_{1}} \frac{\sigma_{n}^{2}}{\sigma_{1}^{2}} & 0 \\
0 & \frac{1}{\beta_{2}} \frac{\sigma_{n}^{2}}{\sigma_{2}^{2}}
\end{array}\right]
\end{aligned}
$$

with $\beta_{k} \stackrel{\text { def }}{=} 2\left(\left\|\mathbf{a}_{k}^{\prime}\right\|^{2}-\gamma_{k}\left(\theta_{1}, \theta_{2}\right)\right), \quad k=1,2$, where $\gamma_{k}\left(\theta_{1}, \theta_{2}\right) \stackrel{\text { def }}{=} \frac{1}{M^{2}-\left|\mathbf{a}_{1}^{H} \mathbf{a}_{2}\right|^{2}} M\left(\left|\mathbf{a}_{k}^{\prime H} \mathbf{a}_{k}\right|^{2}+\left|\mathbf{a}_{k}^{\prime H} \mathbf{a}_{3-k}\right|^{2}\right)-$ $2 \Re\left(\mathbf{a}_{k}^{H} \mathbf{a}_{k}^{\prime} \mathbf{a}_{3-k}^{H} \mathbf{a}_{k} \mathbf{a}_{k}^{\prime}{ }^{H} \mathbf{a}_{3-k}\right), k=1,2$.

If the transmitted symbols are known, the derivation of the FIM $\mathbf{I}_{\mathrm{F}}^{\mathrm{DA}}$ follows the same lines that for the single source case. And more specifically because $\lim _{T \rightarrow \infty} \frac{1}{T} \sum_{t=1}^{T} \epsilon_{t, 1} \epsilon_{t, 2}=\mathrm{E}\left(\epsilon_{t, 1} \epsilon_{t, 2}\right)=0$, the parameter associated with the two sources are decoupled and it is straightforward to prove that the asymptotic (for $T \gg 1)$ FIM $\mathbf{I}_{\mathrm{F}}^{\mathrm{DA}}$ is given by (6) as well.

The second experiment considers two independent and equipowered BPSK or QPSK distributed sources. Fig.4 compares $\operatorname{CRB}_{\mathrm{BPSK}}\left(\theta_{1}\right)$ given by $(5)$ with the $\mathrm{CRB}$ under the non-circular complex Gaussian distribution. And to be fair, this comparison must be done under the same a priori that the two sources are independent with $\left(\rho_{k}=1\right)_{k=1,2}$, i.e., with the same parameter $\boldsymbol{\alpha} \stackrel{\text { def }}{=}\left(\sigma_{n}, \sigma_{1}, \phi_{1}, \theta_{1}, \sigma_{2}, \phi_{2}, \theta_{2}\right)^{T}$. For that reason, we use the non-explicit expression of $\operatorname{CRB}_{\mathrm{NCG}}\left(\theta_{1}\right)$ obtained in [13] which can take this a priori information into account. Fig. 4 exhibits the ratio $\frac{\operatorname{CRB}_{\mathrm{BPSK}}\left(\theta_{1}\right)}{\operatorname{CRB}_{\mathrm{NCG}}\left(\theta_{1}\right)}$ as a function of the DOA separation $\theta_{2}-\theta_{1}$ for three values of the phase separation $\Delta \phi \stackrel{\text { def }}{=} \phi_{2}-\phi_{1}$. We see that the CRB is very sensitive to the phase separation except for large DOA separation. This figure shows, that contrary to the single source case, the CRB under the noncircular complex Gaussian distribution is a very loose upper bound on the CRB under the BPSK distribution except for large values of the DOA and phase separation.

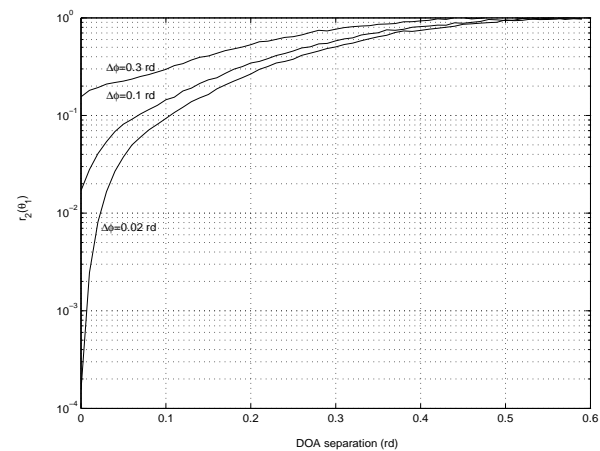

Fig.4 Ratio $r_{2}\left(\theta_{1}\right) \stackrel{\text { def }}{=} \frac{\operatorname{CRB}_{\mathrm{BPSK}}\left(\theta_{1}\right)}{\mathrm{CRB}_{\mathrm{NCG}}\left(\theta_{1}\right)}$ as a function of the DOA separation for different values of the phase separation $\Delta \phi$ for $M=$ 6 and $S N R=20 d B$.

Fig.5 exhibits the domain of validity of the high SNR approximation. We see from this figure that this domain depends not only on $M$, SNR and DOA separation, but also on the distributed sources. It is shown that this domain reduces for QPSK sources compared to BPSK sources. The larger the DOA separation is or the larger $M$ is, the larger the domain of validity of the approximation is.

Since the CRB under the noncircular [resp. circular] Gaussian distribution is a very loose upper bound on the CRB under the discrete BPSK [resp. QPSK] distribution, specifically for small DOA or phase separation, the ML estimators that take these discrete distributions into account outperform the stochastic ML estimator under the circular Gaussian distribution (see e.g., [4]) and the weighed subspace fitting estimator (see e.g., [14]) which both reach $\mathrm{CRB}_{\mathrm{CG}}\left(\theta_{1}\right)$. Consequently, the EM approaches [15] that are iterative procedures capable of implementing the stochastic ML estimator under these discrete distributions outperform the ML estimator under noncircular or circular Gaussian distribution. 


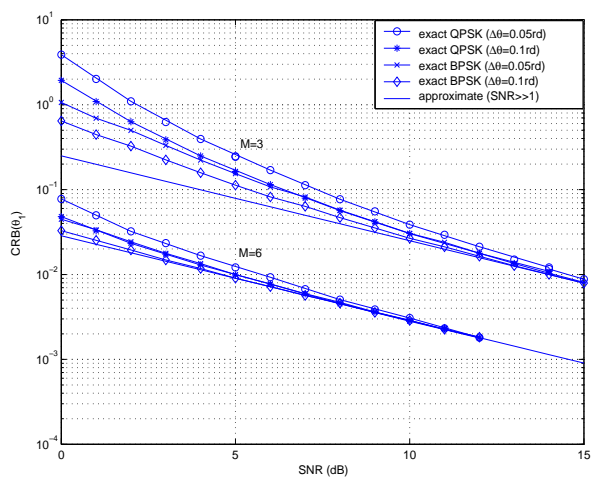

Fig.5 Approximate and exact value (obtained thanks to (5) with $\left.T^{\prime}=10000\right)$ of $\operatorname{CRB}_{\mathrm{BPSK}}\left(\theta_{1}\right)$ and $\operatorname{CRB}_{\mathrm{QPSK}}\left(\theta_{1}\right)$ as a function of the SNR for different values of the DOA separation.

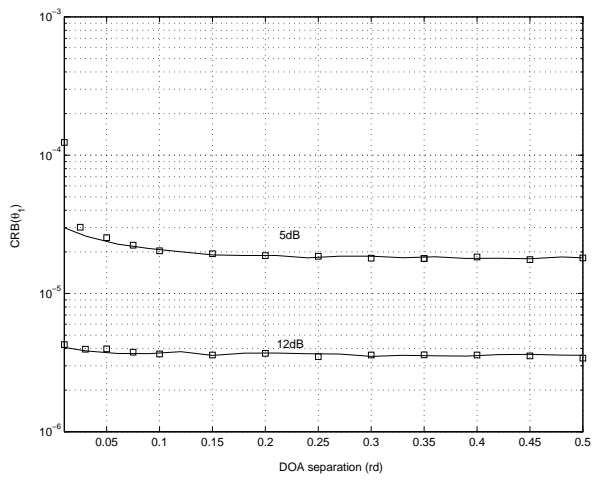

Fig.6 $\operatorname{CRB}_{\mathrm{BPSK}}\left(\theta_{1}\right)$ and estimated $\operatorname{MSE} \mathrm{E}\left(\theta_{1, T}-\theta_{1}\right)^{2}$ given by the deterministic EM algorithm (10 iterations) as a function of the DOA separation for $\Delta \phi=0.1 \mathrm{rd}$.

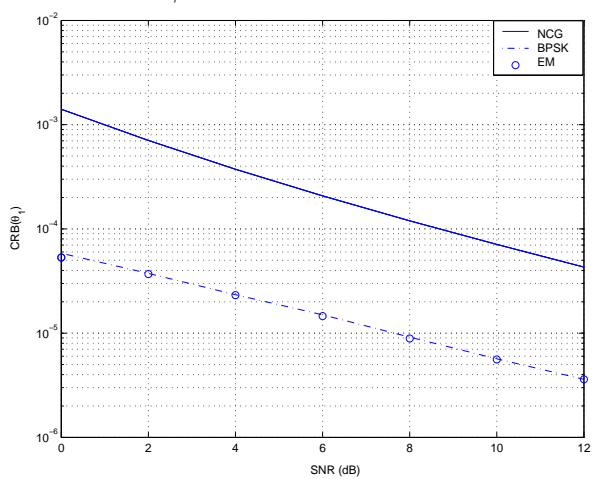

Fig.7 $\operatorname{CRB}_{\mathrm{BPSK}}\left(\theta_{1}\right), \mathrm{CRB}_{\mathrm{NCG}}\left(\theta_{1}\right)$ and estimated $\operatorname{MSE} \mathrm{E}\left(\theta_{1, T}-\right.$ $\left.\theta_{1}\right)^{2}$ given by the deterministic EM algorithm (5 iterations), for $\Delta \theta=0.3 r d$ and $\Delta \phi=0.1 \mathrm{rd}$, versus SNR.

Fig. 6 exhibits $\operatorname{CRB}_{\mathrm{BPSK}}\left(\theta_{1}\right)$ and the estimated mean square error (MSE) $\mathrm{E}\left(\theta_{1, T}-\theta_{1}\right)^{2}$ given by the deterministic EM algorithm initialized by the estimate given by the MUSIC-like algorithm described in [16], as a function of the DOA separation for two SNR's. We see that contrary to $\operatorname{CRB}_{\mathrm{NCG}}\left(\theta_{1}\right), \operatorname{CRB}_{\mathrm{BPSK}}\left(\theta_{1}\right)$ does not increase significantly when decreasing the DOA separation. Fig.7 compares the MSE $\mathrm{E}\left(\theta_{1, T}-\theta_{1}\right)^{2}$ given by the deterministic EM algorithm (initialized as in Fig.6) to $\operatorname{CRB}_{\mathrm{BPSK}}\left(\theta_{1}\right)$ and $\operatorname{CRB}_{\mathrm{NCG}}\left(\theta_{1}\right)$, as a function of the SNR. We see from this figure, that the EM estimate reaches $\operatorname{CRB}_{\mathrm{BPSK}}\left(\theta_{1}\right)$ which largely outperforms $\operatorname{CRB}_{\mathrm{NCG}}\left(\theta_{1}\right)$. To show the asymptotic domain (domain of sample size and SNR for which $\left.\mathrm{E}\left(\theta_{1, T}-\theta_{1}\right)^{2} \approx \operatorname{CRB}_{\mathrm{BPSK}}\left(\theta_{1}\right)\right)$, Figs.8 and 9 compare these CRBs to the estimated mean square error given by the deterministic EM algorithm initialized by values $\theta_{1,0}$ and $\theta_{2,0}$ in the vicinity of $\theta_{1}$ and $\theta_{2}$. We see that the estimates given by the ML estimator under the discrete distribution reach $\operatorname{CRB}_{\mathrm{BPSK}}\left(\theta_{1}\right)$ in a very large domain (from $\mathrm{SNR}=-5 \mathrm{~dB}$ for $T=500$ and from $T=5$ for $\mathrm{SNR}=10 \mathrm{~dB}$ ) in our scenario.

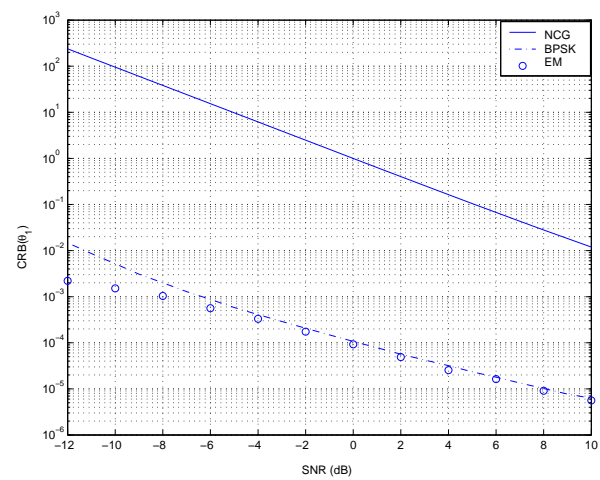

Fig.8 $\operatorname{CRB}_{\mathrm{BPSK}}\left(\theta_{1}\right), \mathrm{CRB}_{\mathrm{NCG}}\left(\theta_{1}\right)$ and estimated (1000 independent runs) mean square error $\mathrm{E}\left(\theta_{1, T}-\theta_{1}\right)^{2}$ given by the deterministic EM algorithm (10 iterations), for $T=500, M=6$, $\Delta \theta=0.05 r d$ and $\Delta \phi=0.1 r d$, versus SNR.

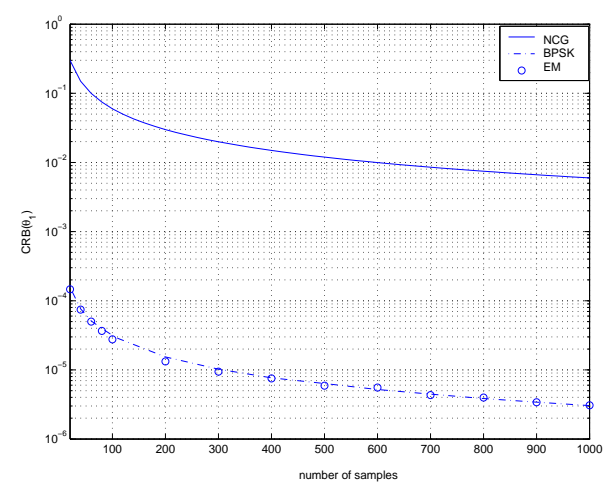

Fig.9 $\operatorname{CRB}_{\mathrm{BPSK}}\left(\theta_{1}\right), \mathrm{CRB}_{\mathrm{NCG}}\left(\theta_{1}\right)$ and estimated (1000 independent runs) mean square error $\mathrm{E}\left(\theta_{1, T}-\theta_{1}\right)^{2}$ given by the deterministic EM algorithm (10 iterations), for $\mathrm{SNR}=10 \mathrm{~d} B, M=6$, $\Delta \theta=0.05 r d$ and $\Delta \phi=0.1 r d$, versus $T$.

\section{CONCLUSION}

This paper developed explicit expressions for the stochastic CRB of the DOA parameter estimates for BPSK and QPSK modulated signals corrupted by additive circular complex Gaussian noise. These stochastic CRB's have been compared, on the one hand, with those obtained with different a priori knowledge, and on the other hand with CRB's under the non-circular and circular complex Gaussian distribution and with different deterministic CRB's.

For a single source, we have proved that the CRB's under the non-circular [resp. circular] complex Gaussian distribution are tight upper bounds on the CRB's under the BPSK [resp. QPSK] distribution at very low and very high SNR only. And for the case of two independent BPSK [resp. QPSK] distributed sources, we have exhibited an important difference of behavior of the stochastic CRB compared to those obtained under the noncircular [resp. circular] Gaussian distribution. Because 
we have proved that the stochastic CRB for the DOA of one source is independent of the parameters of the other source over wide SNR ranges, the CRB under the noncircular [resp. circular] complex Gaussian distribution is a very loose upper bound on the CRB under the BPSK [resp. QPSK] distribution except for large values of the DOA and phase separation. Consequently, ML implementations such as the EM approaches outperform the ML estimator under the circular Gaussian distribution, specifically for small DOA or phase separation.

\section{ApPENDIX}

\section{Appendix: Proof of theorem 1}

The Fisher information matrix is given (elementwise) by:

$$
\frac{1}{T}\left(\mathbf{I}_{F}\right)_{k, l}=-\mathrm{E}\left(\frac{\partial^{2} \ln p\left(\mathbf{y}_{t} ; \boldsymbol{\alpha}\right)}{\partial \alpha_{k} \partial \alpha_{l}}\right) \quad k, l=1, \ldots, 4,
$$

where the PDF (1) are written after straightforward manipulations as

$$
\begin{aligned}
p_{\mathrm{BPSK}}\left(\mathbf{y}_{t} ; \boldsymbol{\alpha}\right) & =\frac{1}{\pi^{M} \sigma_{n}^{2 M}} e^{-\frac{\left\|\mathbf{y}_{t}\right\|^{2}+M \sigma_{1}^{2}}{\sigma_{n}^{2}}} \cosh \left(\frac{\sigma_{1}}{\sigma_{n}^{2}} g_{1}\left(\mathbf{y}_{t}\right)\right) \\
p_{\mathrm{QPSK}}\left(\mathbf{y}_{t} ; \boldsymbol{\alpha}\right) & =\frac{1}{\pi^{M} \sigma_{n}^{2 M}} e^{-\frac{\left\|\mathbf{y}_{t}\right\|^{2}+M \sigma_{1}^{2}}{\sigma_{n}^{2}}} \\
& \cosh \left(\frac{\sigma_{1}}{\sigma_{n}^{2} \sqrt{2}} g_{1}\left(\mathbf{y}_{t}\right)\right) \cosh \left(\frac{\sigma_{1}}{\sigma_{n}^{2} \sqrt{2}} k_{1}\left(\mathbf{y}_{t}\right)\right)
\end{aligned}
$$

with $g_{1}\left(\mathbf{y}_{t}\right) \stackrel{\text { def }}{=} 2 \Re\left(e^{i \phi_{1}} \mathbf{y}_{t}^{H} \mathbf{a}_{1}\right) \quad$ and $\quad k_{1}\left(\mathbf{y}_{t}\right) \stackrel{\text { def }}{=}$ $2 \Im\left(e^{i \phi_{1}} \mathbf{y}_{t}^{H} \mathbf{a}_{1}\right)$.

We evaluate (8) for the BPSK modulation by taking partial derivatives as follows:

$$
\begin{aligned}
\frac{\partial^{2} \ln p\left(\mathbf{y}_{t} ; \boldsymbol{\alpha}\right)}{\partial \sigma_{1}^{2}} & =-\frac{2 M}{\sigma_{n}^{2}}+\frac{g_{1}^{2}\left(\mathbf{y}_{t}\right)}{\sigma_{n}^{4}} \frac{1}{\cosh ^{2}\left(\frac{\sigma_{1} g_{1}\left(\mathbf{y}_{t}\right)}{\sigma_{n}^{2}}\right)} \\
\frac{\partial^{2} \ln p\left(\mathbf{y}_{t} ; \boldsymbol{\alpha}\right)}{\partial \sigma_{1} \partial \sigma_{n}} & =\frac{4 \sigma_{1} M}{\sigma_{n}^{3}}-\frac{2 \sigma_{1} g_{1}^{2}\left(\mathbf{y}_{t}\right)}{\sigma_{n}^{5}} \frac{1}{\cosh ^{2}\left(\frac{\sigma_{1} g_{1}\left(\mathbf{y}_{t}\right)}{\sigma_{n}^{2}}\right)} \\
& -\frac{2 g_{1}\left(\mathbf{y}_{t}\right)}{\sigma_{n}^{3}} \tanh \left(\frac{\sigma_{1} g_{1}\left(\mathbf{y}_{t}\right)}{\sigma_{n}^{2}}\right)
\end{aligned}
$$

$$
\begin{aligned}
& \frac{\partial^{2} \ln p\left(\mathbf{y}_{t} ; \boldsymbol{\alpha}\right)}{\partial \sigma_{n}^{2}}=\frac{2 \sigma_{n}^{2} M-6\left(\sigma_{1}^{2} M+\left|\mathbf{y}_{t}\right|^{2}\right)}{\sigma_{n}^{4}} \\
& +\frac{4 \sigma_{1}^{2} g_{1}^{2}\left(\mathbf{y}_{t}\right)}{\sigma_{n}^{6}} \frac{1}{\cosh ^{2}\left(\frac{\sigma_{1} g_{1}\left(\mathbf{y}_{t}\right)}{\sigma_{n}^{2}}\right)} \\
& +\frac{6 \sigma_{1} g_{1}\left(\mathbf{y}_{t}\right)}{\sigma_{n}^{4}} \tanh \left(\frac{\sigma_{1} g_{1}\left(\mathbf{y}_{t}\right)}{\sigma_{n}^{2}}\right) \\
& \frac{\partial^{2} \ln p\left(\mathbf{y}_{t} ; \boldsymbol{\alpha}\right)}{\partial \phi_{1}^{2}}=\frac{\sigma_{1}^{2} k_{1}^{2}\left(\mathbf{y}_{t}\right)}{\sigma_{n}^{4}} \frac{1}{\cosh ^{2}\left(\frac{\sigma_{1} g_{1}\left(\mathbf{y}_{t}\right)}{\sigma_{n}^{2}}\right)} \\
& -\frac{\sigma_{1} g_{1}\left(\mathbf{y}_{t}\right)}{\sigma_{n}^{2}} \tanh \left(\frac{\sigma_{1} g_{1}\left(\mathbf{y}_{t}\right)}{\sigma_{n}^{2}}\right) \\
& \frac{\partial^{2} \ln p\left(\mathbf{y}_{t} ; \boldsymbol{\alpha}\right)}{\partial \sigma_{1} \partial \phi_{1}}=-\frac{\sigma_{1} g_{1}\left(\mathbf{y}_{t}\right) k_{1}\left(\mathbf{y}_{t}\right)}{\sigma_{n}^{4}} \frac{1}{\cosh ^{2}\left(\frac{\sigma_{1} g_{1}\left(\mathbf{y}_{t}\right)}{\sigma_{n}^{2}}\right)} \\
& -\frac{k_{1}\left(\mathbf{y}_{t}\right)}{\sigma_{n}^{2}} \tanh \left(\frac{\sigma_{1} g_{1}\left(\mathbf{y}_{t}\right)}{\sigma_{n}^{2}}\right) \\
& \frac{\partial^{2} \ln p\left(\mathbf{y}_{t} ; \boldsymbol{\alpha}\right)}{\partial \sigma_{n} \partial \phi_{1}}=\frac{2 \sigma_{1}^{2} g_{1}\left(\mathbf{y}_{t}\right) k_{1}\left(\mathbf{y}_{t}\right)}{\sigma_{n}^{5}} \frac{1}{\cosh ^{2}\left(\frac{\sigma_{1} g_{1}\left(\mathbf{y}_{t}\right)}{\sigma_{n}^{2}}\right)} \\
& +\frac{2 \sigma_{1} k_{1}\left(\mathbf{y}_{t}\right)}{\sigma_{n}^{3}} \tanh \left(\frac{\sigma_{1} g_{1}\left(\mathbf{y}_{t}\right)}{\sigma_{n}^{2}}\right) \\
& \frac{\partial^{2} \ln p\left(\mathbf{y}_{t} ; \boldsymbol{\alpha}\right)}{\partial \sigma_{1} \partial \theta_{1}}=\frac{\sigma_{1} g_{1}\left(\mathbf{y}_{t}\right) g_{1}^{\prime}\left(\mathbf{y}_{t}\right)}{\sigma_{n}^{4}} \frac{1}{\cosh ^{2}\left(\frac{\sigma_{1} g_{1}\left(\mathbf{y}_{t}\right)}{\sigma_{n}^{2}}\right)} \\
& +\frac{g_{1}^{\prime}\left(\mathbf{y}_{t}\right)}{\sigma_{n}^{2}} \tanh \left(\frac{\sigma_{1} g_{1}\left(\mathbf{y}_{t}\right)}{\sigma_{n}^{2}}\right) \\
& \frac{\partial^{2} \ln p\left(\mathbf{y}_{t} ; \boldsymbol{\alpha}\right)}{\partial \sigma_{n} \partial \theta_{1}}=-\frac{2 \sigma_{1}^{2} g_{1}\left(\mathbf{y}_{t}\right) g_{1}^{\prime}\left(\mathbf{y}_{t}\right)}{\sigma_{n}^{5}} \frac{1}{\cosh ^{2}\left(\frac{\sigma_{1} g_{1}\left(\mathbf{y}_{t}\right)}{\sigma_{n}^{2}}\right)} \\
& -\frac{2 \sigma_{1} g_{1}^{\prime}\left(\mathbf{y}_{t}\right)}{\sigma_{n}^{3}} \tanh \left(\frac{\sigma_{1} g_{1}\left(\mathbf{y}_{t}\right)}{\sigma_{n}^{3}}\right) \\
& \frac{\partial^{2} \ln p\left(\mathbf{y}_{t} ; \boldsymbol{\alpha}\right)}{\partial \theta_{1}^{2}}=\frac{\sigma_{1}^{2} g_{1}^{\prime 2}\left(\mathbf{y}_{t}\right)}{\sigma_{n}^{4}} \frac{1}{\cosh ^{2}\left(\frac{\sigma_{1} g_{1}\left(\mathbf{y}_{t}\right)}{\sigma_{n}^{2}}\right)} \\
& +\frac{\sigma_{1} g_{1}^{\prime \prime}\left(\mathbf{y}_{t}\right)}{\sigma_{n}^{2}} \tanh \left(\frac{\sigma_{1} g_{1}\left(\mathbf{y}_{t}\right)}{\sigma_{n}^{2}}\right) \\
& \frac{\partial^{2} \ln p\left(\mathbf{y}_{t} ; \boldsymbol{\alpha}\right)}{\partial \theta_{1} \partial \phi_{1}}=-\frac{\sigma_{1}^{2} g_{1}^{\prime}\left(\mathbf{y}_{t}\right) k_{1}\left(\mathbf{y}_{t}\right)}{\sigma_{n}^{4}} \frac{1}{\cosh ^{2}\left(\frac{\sigma_{1} g_{1}\left(\mathbf{y}_{t}\right)}{\sigma_{n}^{2}}\right)} \\
& -\frac{\sigma_{1} k_{1}^{\prime}\left(\mathbf{y}_{t}\right)}{\sigma_{n}^{2}} \tanh \left(\frac{\sigma_{1} g_{1}\left(\mathbf{y}_{t}\right)}{\sigma_{n}^{2}}\right)
\end{aligned}
$$

with $g_{1}^{\prime}\left(\mathbf{y}_{t}\right) \stackrel{\text { def }}{=} 2 \Re\left(e^{i \phi_{1}} \mathbf{y}_{t}^{H} \mathbf{a}_{1}^{\prime}\right), g_{1}^{\prime \prime}\left(\mathbf{y}_{t}\right) \stackrel{\text { def }}{=} 2 \Re\left(e^{i \phi_{1}} \mathbf{y}_{t}^{H} \mathbf{a}_{1}^{\prime \prime}\right)$ and $k_{1}^{\prime}\left(\mathbf{y}_{t}\right) \stackrel{\text { def }}{=} 2 \Im\left(e^{i \phi_{1}} \mathbf{y}_{t}^{H} \mathbf{a}_{1}^{\prime}\right)$.

Using the regularity condition $\frac{\partial}{\partial \alpha_{k}} \int p(\mathbf{y} ; \boldsymbol{\alpha}) d \mathbf{y}=$ $\int \frac{\partial p(\mathbf{y} ; \boldsymbol{\alpha})}{\partial \alpha_{k}} d \mathbf{y}$ (see e.g., [17, rel. (a.9)]) which is fulfilled for finite mixtures of Gaussian distributions, the following property holds: $\mathrm{E}\left(\frac{\partial \ln p\left(\mathbf{y}_{t} ; \boldsymbol{\alpha}\right)}{\partial \sigma_{1}}\right)=0$. With 
$\frac{\partial \ln p\left(\mathbf{y}_{t} ; \boldsymbol{\alpha}\right)}{\partial \sigma_{1}}=-\frac{2 M \sigma_{1}}{\sigma_{n}^{2}}+\frac{g_{1}\left(\mathbf{y}_{t}\right)}{\sigma_{n}^{2}} \tanh \left(\frac{\sigma_{1} g_{1}\left(\mathbf{y}_{t}\right)}{\sigma_{n}^{2}}\right)$, we obtain

$$
\mathrm{E}\left(g_{1}\left(\mathbf{y}_{t}\right) \tanh \left(\frac{\sigma_{1} g_{1}\left(\mathbf{y}_{t}\right)}{\sigma_{n}^{2}}\right)\right)=2 M \sigma_{1}
$$

This identity enables us to straightforwardly derive the terms $\left(\mathbf{I}_{\mathrm{F}}\right)_{\left(\sigma_{n}, \sigma_{n}\right)},\left(\mathbf{I}_{\mathrm{F}}\right)_{\left(\sigma_{n}, \sigma_{1}\right)}$ and $\left(\mathbf{I}_{\mathrm{F}}\right)_{\left(\sigma_{1}, \sigma_{1}\right)}$ of $\mathbf{I}_{\mathrm{F}}^{\mathrm{BPSK}}$ thanks to the definition of the function $f_{1}(\rho)$.

To evaluate $\left(\mathbf{I}_{\mathrm{F}}\right)_{\left(\phi_{1}, \phi_{1}\right)}$, we note that $g_{1}\left(\mathbf{y}_{t}\right)=$ $2 M \sigma_{1} \epsilon_{t}+\left(e^{i \phi_{1}} \mathbf{n}_{t}^{H} \mathbf{a}_{1}+e^{-i \phi_{1}} \mathbf{a}_{1}^{H} \mathbf{n}_{t}\right)$ and $k_{1}\left(\mathbf{y}_{t}\right)=$ $\frac{1}{i}\left(e^{i \phi_{1}} \mathbf{n}_{t}^{H} \mathbf{a}_{1}-e^{-i \phi_{1}} \mathbf{a}_{1}^{H} \mathbf{n}_{t}\right)$. Because $\epsilon_{t}$ and the couple $\left(e^{i \phi_{1}} \mathbf{n}_{t}^{H} \mathbf{a}_{1}+e^{-i \phi_{1}} \mathbf{a}_{1}^{H} \mathbf{n}_{t}, \frac{1}{i}\left(e^{i \phi_{1}} \mathbf{n}_{t}^{H} \mathbf{a}_{1}-e^{-i \phi_{1}} \mathbf{a}_{1}^{H} \mathbf{n}_{t}\right)\right)$ are independent, and that these last two Gaussian random variables are uncorrelated and consequently independent, the three random variables $\epsilon_{t}, e^{i \phi_{1}} \mathbf{n}_{t}^{H} \mathbf{a}_{1}+$ $e^{-i \phi_{1}} \mathbf{a}_{1}^{H} \mathbf{n}_{t}$ and $\frac{1}{i}\left(e^{i \phi_{1}} \mathbf{n}_{t}^{H} \mathbf{a}_{1}-e^{-i \phi_{1}} \mathbf{a}_{1}^{H} \mathbf{n}_{t}\right)$ are collectively independent and thus $g_{1}\left(\mathbf{y}_{t}\right)$ and $k_{1}\left(\mathbf{y}_{t}\right)$ are independent. Therefore

$$
\begin{aligned}
\mathrm{E}\left(\frac{k_{1}^{2}\left(\mathbf{y}_{t}\right)}{\cosh ^{2}\left(\frac{\sigma_{1} g_{1}\left(\mathbf{y}_{t}\right)}{\sigma_{n}^{2}}\right)}\right)= & \mathrm{E}\left(k_{1}^{2}\left(\mathbf{y}_{t}\right)\right) \\
& \mathrm{E}\left(\frac{1}{\cosh ^{2}\left(\frac{\sigma_{1} g_{1}\left(\mathbf{y}_{t}\right)}{\sigma_{n}^{2}}\right)}\right)
\end{aligned}
$$

with $\mathrm{E}\left(k_{1}^{2}\left(\mathbf{y}_{t}\right)\right)=2 M \sigma_{n}^{2}$. With $\mathrm{E}\left(\frac{1}{\cosh ^{2}\left(\frac{\sigma_{1} g_{1}\left(\mathbf{y}_{t}\right)}{\sigma_{n}^{2}}\right)}\right)=$ $f_{2}(\rho)$ and thanks to the identity $(9),\left(\mathbf{I}_{\mathrm{F}}\right)_{\left(\phi_{1}, \phi_{1}\right)}$ is straightforwardly derived.

Noting that $g_{1}^{\prime}\left(\mathbf{y}_{t}\right)=e^{i \phi_{1}} \mathbf{n}_{t}^{H} \mathbf{a}_{1}^{\prime}+e^{-i \phi_{1}} \mathbf{a}_{1}^{\prime}{ }^{H} \mathbf{n}_{t}$, thanks to $\mathbf{a}_{1}^{\prime}{ }^{H} \mathbf{a}_{1}+\mathbf{a}_{1}^{H} \mathbf{a}_{1}^{\prime}=0$ derived from $\left\|\mathbf{a}_{1}\right\|^{2}=M$. Consequently for the same reason that $g_{1}\left(\mathbf{y}_{t}\right)$ and $k_{1}\left(\mathbf{y}_{t}\right)$, $g_{1}\left(\mathbf{y}_{t}\right)$ and $g_{1}^{\prime}\left(\mathbf{y}_{t}\right)$ are independent as well. And because $k_{1}\left(\mathbf{y}_{t}\right)$ and $g_{1}^{\prime}\left(\mathbf{y}_{t}\right)$ are zero-mean, the expectations of the two terms of $\frac{\partial^{2} \ln p\left(\mathbf{y}_{t} ; \boldsymbol{\alpha}\right)}{\partial \sigma_{1} \partial \phi_{1}}, \frac{\partial^{2} \ln p\left(\mathbf{y}_{t} ; \boldsymbol{\alpha}\right)}{\partial \sigma_{n} \partial \phi_{1}}, \frac{\partial^{2} \ln p\left(\mathbf{y}_{t} ; \boldsymbol{\alpha}\right)}{\partial \sigma_{1} \partial \theta_{1}}$ and $\frac{\partial^{2} \ln p\left(\mathbf{y}_{t} ; \boldsymbol{\alpha}\right)}{\partial \sigma_{n} \partial \theta_{1}}$ vanish and therefore $\left(\mathbf{I}_{\mathrm{F}}\right)_{\left(\sigma_{1}, \phi_{1}\right)}=$ $\left(\mathbf{I}_{\mathrm{F}}\right)_{\left(\sigma_{n}, \phi_{1}\right)}=\left(\mathbf{I}_{\mathrm{F}}\right)_{\left(\sigma_{1}, \theta_{1}\right)}=\left(\mathbf{I}_{\mathrm{F}}\right)_{\left(\sigma_{n}, \theta_{1}\right)}=0$. way

Considering the first term of $\frac{\partial^{2} \ln p\left(\mathbf{y}_{t} ; \boldsymbol{\alpha}\right)}{\partial \theta_{1}^{2}}$, in the same

$$
\begin{aligned}
\mathrm{E}\left(\frac{g_{1}^{\prime 2}\left(\mathbf{y}_{t}\right)}{\cosh ^{2}\left(\frac{\sigma_{1} g_{1}\left(\mathbf{y}_{t}\right)}{\sigma_{n}^{2}}\right)}\right)= & \mathrm{E}\left(g_{1}^{\prime 2}\left(\mathbf{y}_{t}\right)\right) \\
& \mathrm{E}\left(\frac{1}{\cosh ^{2}\left(\frac{\sigma_{1} g_{1}\left(\mathbf{y}_{t}\right)}{\sigma_{n}^{2}}\right)}\right)
\end{aligned}
$$

With $\mathrm{E}\left(g_{1}^{\prime 2}\left(\mathbf{y}_{t}\right)\right)=2\left\|\mathbf{a}_{1}^{\prime}\right\|^{2} \sigma_{n}^{2}$.

For the first term of $\frac{\partial^{2} \ln p\left(\mathbf{y}_{t} ; \boldsymbol{\alpha}\right)}{\partial \theta_{1} \partial \phi_{1}}$, we note that the random variables $\epsilon_{t}$ and

$\left(e^{i \phi_{1}} \mathbf{n}_{t}^{H} \mathbf{a}_{1}+e^{-i \phi_{1}} \mathbf{a}_{1}^{H} \mathbf{n}_{t},\left(g_{1}^{\prime}\left(\mathbf{y}_{t}\right), k_{1}\left(\mathbf{y}_{t}\right)\right)\right)$ are independent. And since the zero-mean Gaussian random variables $e^{i \phi_{1}} \mathbf{n}_{t}^{H} \mathbf{a}_{1}+e^{-i \phi_{1}} \mathbf{a}_{1}^{H} \mathbf{n}_{t}$ and $\left(g_{1}^{\prime}\left(\mathbf{y}_{t}\right), k_{1}\left(\mathbf{y}_{t}\right)\right)$ are independent too, the three random variables $\epsilon_{t}$, $e^{i \phi_{1}} \mathbf{n}_{t}^{H} \mathbf{a}_{1}+e^{-i \phi_{1}} \mathbf{a}_{1}^{H} \mathbf{n}_{t}$ and $\left(g_{1}^{\prime}\left(\mathbf{y}_{t}\right), k_{1}\left(\mathbf{y}_{t}\right)\right)$ are collectively independent. Consequently the sum $g_{1}\left(\mathbf{y}_{t}\right)$ of these first two random variables is independent of the last random variable $\left(g_{1}^{\prime}\left(\mathbf{y}_{t}\right), k_{1}\left(\mathbf{y}_{t}\right)\right)$ and thus

$$
\begin{array}{r}
\mathrm{E}\left(\frac{g_{1}^{\prime}\left(\mathbf{y}_{t}\right) k_{1}\left(\mathbf{y}_{t}\right)}{\cosh ^{2}\left(\frac{\sigma_{1} g_{1}\left(\mathbf{y}_{t}\right)}{\sigma_{n}^{2}}\right)}\right)= \\
\mathrm{E}\left(g_{1}^{\prime}\left(\mathbf{y}_{t}\right) k_{1}\left(\mathbf{y}_{t}\right)\right) \\
\mathrm{E}\left(\frac{1}{\cosh ^{2}\left(\frac{\sigma_{1} g_{1}\left(\mathbf{y}_{t}\right)}{\sigma_{n}^{2}}\right)}\right)
\end{array}
$$

with $\mathrm{E}\left(g_{1}^{\prime}\left(\mathbf{y}_{t}\right) k_{1}\left(\mathbf{y}_{t}\right)\right)=-2 \sigma_{n}^{2}\left(i \mathbf{a}_{1}^{\prime}{ }^{H} \mathbf{a}_{1}\right)$ (see Section 4).

Finally, regarding the second term of $\frac{\partial^{2} \ln p\left(\mathbf{y}_{t} ; \boldsymbol{\alpha}\right)}{\partial \theta_{1}^{2}}$ and $\frac{\partial^{2} \ln p\left(\mathbf{y}_{t} ; \boldsymbol{\alpha}\right)}{\partial \theta_{1} \partial \phi_{1}}$, we have to elaborate a little bit. Because $g_{1}^{\prime \prime}\left(\mathbf{y}_{t}\right)=-2\left\|\mathbf{a}_{1}^{\prime}\right\|^{2} \sigma_{1} \epsilon_{t}+x_{t}^{\prime \prime}$ with $x_{t}^{\prime \prime} \stackrel{\text { def }}{=}$ $e^{i \phi_{1}} \mathbf{n}_{t}^{H} \mathbf{a}_{1}^{\prime \prime}+e^{-i \phi_{1}} \mathbf{a}_{1}^{\prime \prime} \mathbf{n}_{t}$ and $g_{1}\left(\mathbf{y}_{t}\right)=2 M \sigma_{1} \epsilon_{t}+x_{t}$ with $x_{t} \stackrel{\text { def }}{=} e^{i \phi_{1}} \mathbf{n}_{t}^{H} \mathbf{a}_{1}+e^{-i \phi_{1}} \mathbf{a}_{1}^{H} \mathbf{n}_{t}$ where $\left(x_{t}^{\prime \prime}, x_{t}\right)$ is zero-mean Gaussian distributed, we have ${ }^{4}$

$g_{1}^{\prime \prime}\left(\mathbf{y}_{t}\right)=-\frac{\left\|\mathbf{a}_{1}^{\prime}\right\|^{2}}{M} g_{1}\left(\mathbf{y}_{t}\right)+x_{t}^{\prime} \quad$ with $\quad x_{t}^{\prime} \stackrel{\text { def }}{=} x_{t}^{\prime \prime}+\frac{\left\|\mathbf{a}_{1}^{\prime}\right\|^{2}}{M} x_{t}$

where $\left(x_{t}, x_{t}^{\prime}\right)$ is zero-mean Gaussian distributed with $\mathrm{E}\left(x_{t} x_{t}^{\prime}\right)=\mathrm{E}\left(x_{t} x_{t}^{\prime \prime}\right)+\frac{\left\|\mathbf{a}_{1}^{\prime}\right\|^{2}}{M} \mathrm{E}\left(x_{t}^{2}\right)=-2\left\|\mathbf{a}_{1}^{\prime}\right\|^{2} \sigma_{n}^{2}+$ $\frac{\left\|\mathbf{a}_{1}^{\prime}\right\|^{2}}{M}\left(2 M \sigma_{n}^{2}\right)=0$ and thus $x_{t}$ and $x_{t}^{\prime}$ are independent. Because $g_{1}\left(\mathbf{y}_{t}\right)=2 M \sigma_{1} \epsilon_{t}+x_{t}$ where the discrete random variable $\epsilon_{t}$ is independent of the noise random variables $x_{t}{ }^{\prime}$, the random variables $g_{1}\left(\mathbf{y}_{t}\right)$ and $x_{t}^{\prime}$ are independent and then

$$
\begin{aligned}
\mathrm{E}\left(g_{1}^{\prime \prime}\left(\mathbf{y}_{t}\right) \tanh \left(\frac{\sigma_{1} g_{1}\left(\mathbf{y}_{t}\right)}{\sigma_{n}^{2}}\right)\right) \\
=\mathrm{E}\left(-\frac{\left\|\mathbf{a}_{1}^{\prime}\right\|^{2}}{M} g_{1}\left(\mathbf{y}_{t}\right) \tanh \left(\frac{\sigma_{1} g_{1}\left(\mathbf{y}_{t}\right)}{\sigma_{n}^{2}}\right)\right) \\
+\mathrm{E}\left(x_{t}^{\prime}\right) \mathrm{E}\left(\tanh \left(\frac{\sigma_{1} g_{1}\left(\mathbf{y}_{t}\right)}{\sigma_{n}^{2}}\right)\right) \\
=-\frac{\left\|\mathbf{a}_{1}^{\prime}\right\|^{2}}{M} \mathrm{E}\left(g_{1}\left(\mathbf{y}_{t}\right) \tanh \left(\frac{\sigma_{1} g_{1}\left(\mathbf{y}_{t}\right)}{\sigma_{n}^{2}}\right)\right)
\end{aligned}
$$

and $\left(\mathbf{I}_{\mathrm{F}}\right)_{\left(\theta_{1}, \theta_{1}\right)}$ follows from identity (9). The same approach applies to evaluate $\mathrm{E}\left(k_{1}^{\prime}\left(\mathbf{y}_{t}\right) \tanh \left(\frac{\sigma_{1} g_{1}\left(\mathbf{y}_{t}\right)}{\sigma_{n}^{2}}\right)\right)$ and gives the term $\left(\mathbf{I}_{\mathrm{F}}\right)_{\left(\theta_{1}, \phi_{1}\right)}$.

For the QPSK modulation, evaluating the partial derivatives $\frac{\partial^{2} \ln p\left(\mathbf{y}_{t} ; \boldsymbol{\alpha}\right)}{\partial \alpha_{k} \partial \alpha_{l}}$ and taking their expectation are derived in the same way, provided the log-likelihoods

${ }^{4}$ Because $\left\|\mathbf{a}_{1}\right\|^{2}=M$ implies $\frac{d^{2}\left\|\mathbf{a}_{1}\right\|^{2}}{d \theta_{1}^{2}}=\mathbf{a}_{1}^{H} \mathbf{a}_{1}^{\prime \prime}+\mathbf{a}_{1}^{\prime \prime} \mathbf{a}_{1}+$ $2\left\|\mathbf{a}_{1}^{\prime}\right\|^{2}=0$. 
associated with $g_{1}\left(\mathbf{y}_{t}\right)$ and $k_{1}\left(\mathbf{y}_{t}\right)$ are gathered as well as the hypothesis of independence of $\Re\left(\epsilon_{t}\right)$ and $\Im\left(\epsilon_{t}\right)$ is taken into account.

\section{Appendix: Proof of (6)}

The derivation of (6) results from the following alternative form of the FIM:

$$
\frac{1}{T}\left(\mathbf{I}_{F}\right)_{k, l}=\mathrm{E}\left(\frac{\partial \ln p\left(\mathbf{y}_{t} ; \boldsymbol{\alpha}\right)}{\partial \alpha_{k}} \frac{\partial \ln p\left(\mathbf{y}_{t} ; \boldsymbol{\alpha}\right)}{\partial \alpha_{l}}\right)
$$

$k, l=1, \ldots, 7$.

We consider only the terms of (10) associated with $\phi_{1}$ and $\phi_{2}$ because the other terms follow along the same lines. For the BPSK derivation, the PDF of $\mathbf{y}_{t}$ is a mixture of 4 Gaussian PDFs and

$$
\frac{\partial \ln p\left(\mathbf{y}_{t} ; \boldsymbol{\alpha}\right)}{\partial \phi_{k}}=-\frac{1}{\sigma_{n}^{2}} \frac{\sum_{j=1}^{4} \frac{\partial h_{j}}{\partial \phi_{k}} e^{-\frac{h_{j}}{\sigma_{n}^{2}}}}{\sum_{j=1}^{4} e^{-\frac{h_{j}}{\sigma_{n}^{2}}}} \quad k=1,2 .
$$

with

$$
\begin{aligned}
& h_{1}=g_{1}+g_{2}+g_{1,2}, h_{2}=g_{1}-g_{2}-g_{1,2}, \\
& h_{3}=-g_{1}+g_{2}-g_{1,2} \text { and } h_{4}=-g_{1}-g_{2}+g_{1,2}
\end{aligned}
$$

where the random variable $\left(g_{k}\right)_{k=1,2}$ are defined by $g_{k} \stackrel{\text { def }}{=}$ $2 \Re\left(e^{i \phi_{k}} \mathbf{y}_{t}^{H} \mathbf{a}_{k}\right)=\sigma_{k}\left(e^{i \phi_{k}} \mathbf{y}_{t}^{H} \mathbf{a}_{k}+e^{-i \phi_{k}} \mathbf{a}_{k}^{H} \mathbf{y}_{t}\right)$ with $\mathbf{y}_{t}=$ $\sigma_{1} e^{i \phi_{1}} \epsilon_{t, 1} \mathbf{a}_{1}+\sigma_{2} e^{i \phi_{2}} \epsilon_{t, 2} \mathbf{a}_{2}+\mathbf{n}_{t}, k=1,2$ and $g_{1,2} \stackrel{\text { def }}{=}$ $\sigma_{1} \sigma_{2}\left(e^{i\left(\phi_{1}-\phi_{2}\right)} \mathbf{a}_{2}^{H} \mathbf{a}_{1}+e^{-i\left(\phi_{1}-\phi_{2}\right)} \mathbf{a}_{1}^{H} \mathbf{a}_{2}\right)$.

Because the random variables $\left(\epsilon_{t, 1}, \epsilon_{t, 2}\right)$ and $\mathbf{n}_{t}$ are independent, we can condition the random variable $\frac{\partial \ln p\left(\mathbf{y}_{t} ; \boldsymbol{\alpha}\right)}{\partial \alpha_{t}} \frac{\partial \ln p\left(\mathbf{y}_{t} ; \boldsymbol{\alpha}\right)}{\partial \alpha_{l}}$ w.r.t. the different couples $\left(\epsilon_{t, 1}, \epsilon_{t, 2}\right)=\left(\eta_{l, 1}, \eta_{l, 2}\right)_{l=1,4}$ of symboles to compute the expectation (10). In the following, we prove, that among the four exponentials in (11), three of them are insignificant w.r.t. one of them that is dominant. For example, for $\left(\eta_{l, 1}, \eta_{l, 2}\right)_{l=1}=(-1,-1)$, we have for $k=1,2$

$$
g_{k}=-2 M \sigma_{k}^{2}-g_{1,2}+\sigma_{k}\left(e^{i \phi_{k}} \mathbf{n}_{t}^{H} \mathbf{a}_{k}+e^{-i \phi_{k}} \mathbf{a}_{k}^{H} \mathbf{n}_{t}\right),
$$

and for $M \frac{\sigma_{1}^{2}}{\sigma_{n}^{2}} \gg 1$ and $M \frac{\sigma_{2}^{2}}{\sigma_{n}^{2}} \gg 1$ :

$$
\begin{aligned}
-\frac{h_{1}}{\sigma_{n}^{2}} & =\frac{2 M\left(\sigma_{1}^{2}+\sigma_{2}^{2}\right)}{\sigma_{n}^{2}}-n_{1}^{\prime}+\frac{g_{1,2}}{\sigma_{n}^{2}} \\
& \approx \frac{2 M\left(\sigma_{1}^{2}+\sigma_{2}^{2}\right)}{\sigma_{n}^{2}}+\frac{g_{1,2}}{\sigma_{n}^{2}} \\
-\frac{h_{2}}{\sigma_{n}^{2}} & =\frac{2 M\left(\sigma_{1}^{2}-\sigma_{2}^{2}\right)}{\sigma_{n}^{2}}-n_{2}^{\prime}+\frac{g_{1,2}}{\sigma_{n}^{2}} \\
& \approx \frac{2 M\left(\sigma_{1}^{2}-\sigma_{2}^{2}\right)}{\sigma_{n}^{2}}+\frac{g_{1,2}}{\sigma_{n}^{2}} \\
-\frac{h_{3}}{\sigma_{n}^{2}} & =\frac{2 M\left(\sigma_{2}^{2}-\sigma_{1}^{2}\right)}{\sigma_{n}^{2}}+n_{2}^{\prime}+\frac{g_{1,2}}{\sigma_{n}^{2}} \\
& \approx \frac{2 M\left(\sigma_{2}^{2}-\sigma_{1}^{2}\right)}{\sigma_{n}^{2}}+\frac{g_{1,2}}{\sigma_{n}^{2}}
\end{aligned}
$$

$$
\begin{aligned}
-\frac{h_{4}}{\sigma_{n}^{2}} & =-\frac{2 M\left(\sigma_{1}^{2}+\sigma_{2}^{2}\right)}{\sigma_{n}^{2}}+n_{1}^{\prime}-\frac{3 g_{1,2}}{\sigma_{n}^{2}} \\
& \approx-\frac{2 M\left(\sigma_{1}^{2}+\sigma_{2}^{2}\right)}{\sigma_{n}^{2}}-\frac{3 g_{1,2}}{\sigma_{n}^{2}}
\end{aligned}
$$

where $\left(n_{k}^{\prime}\right)_{k=1,2}$ are zero-mean Gaussian random variables of variance

$$
\begin{array}{r}
\frac{2}{\sigma_{n}^{2}}\left(M\left(\sigma_{1}^{2}+\sigma_{2}^{2}\right)-(-1)^{k} \Re\left(\sigma_{1} \sigma_{2} e^{i\left(\phi_{1}-\phi_{2}\right)} \mathbf{a}_{1}^{H} \mathbf{a}_{2}\right)\right) \\
>\frac{2 M\left(\sigma_{1}^{2}+\sigma_{2}^{2}-\sigma_{1} \sigma_{2}\right)}{\sigma_{n}^{2}} .
\end{array}
$$

Consequently

$$
\begin{aligned}
-\frac{h_{1}}{\sigma_{n}^{2}}-\left(-\frac{h_{2}}{\sigma_{n}^{2}}\right) & \approx \frac{2 M \sigma_{2}^{2}}{\sigma_{n}^{2}} \gg 1 \\
-\frac{h_{1}}{\sigma_{n}^{2}}-\left(-\frac{h_{3}}{\sigma_{n}^{2}}\right) & \approx \frac{2 M \sigma_{1}^{2}}{\sigma_{n}^{2}} \gg 1 \\
-\frac{h_{1}}{\sigma_{n}^{2}}-\left(-\frac{h_{4}}{\sigma_{n}^{2}}\right) & \approx \frac{4 M\left(\sigma_{1}^{2}+\sigma_{2}^{2}\right)}{\sigma_{n}^{2}}+\frac{4 g_{1,2}}{\sigma_{n}^{2}} \\
& \geq \frac{4 M\left(\sigma_{1}^{2}+\sigma_{2}^{2}-2 \alpha \sigma_{1} \sigma_{2}\right)}{\sigma_{n}^{2}} \\
& \geq \frac{8 M \sigma_{1} \sigma_{2}(1-\alpha)}{\sigma_{n}^{2}} \\
& \geq \frac{8 M(1-\alpha) \operatorname{Inf}\left(\sigma_{1}^{2}, \sigma_{2}^{2}\right)}{\sigma_{n}^{2}} \gg 1
\end{aligned}
$$

where $\alpha$ defined by $\operatorname{Inf}\left(g_{1,2}\right)=-2 M \alpha \sigma_{1} \sigma_{2}$ is related to the height of first sidelobe of the beam pattern of the array and satisfies for all standard array $\alpha<0.5^{5}$. Therefore it is proved that the term $e^{-\frac{h_{1}}{\sigma_{n}^{2}}}$ is dominant w.r.t. the terms $e^{-\frac{h_{2}}{\sigma_{n}^{2}}}, e^{-\frac{h_{3}}{\sigma_{n}^{2}}}$ and $e^{-\frac{h_{4}}{\sigma_{n}^{2}}}$ and

$$
\begin{aligned}
\frac{\partial \ln p\left(\mathbf{y}_{t} ; \boldsymbol{\alpha}\right)}{\partial \phi_{k}} & \approx-\frac{1}{\sigma_{n}^{2}} \frac{\partial h_{1}}{\partial \phi_{k}}=-\frac{1}{\sigma_{n}^{2}}\left(\frac{\partial g_{k}}{\partial \phi_{k}}+\frac{\partial g_{1,2}}{\partial \phi_{k}}\right) \\
& =-\frac{i \sigma_{k}}{\sigma_{n}^{2}}\left(e^{i \phi_{k}} \mathbf{n}_{t}^{H} \mathbf{a}_{k}-e^{-i \phi_{k}} \mathbf{a}_{k}^{H} \mathbf{n}_{t}\right)
\end{aligned}
$$

and for an arbitrary couple of symbols:

$$
\begin{aligned}
& \frac{\partial \ln p\left(\mathbf{y}_{t} ; \boldsymbol{\alpha}\right)}{\partial \phi_{k}} \approx-\frac{i \sigma_{k}}{\sigma_{n}^{2}}\left(e^{i \phi_{k}} \mathbf{n}_{t}^{H} \mathbf{a}_{k}-e^{-i \phi_{k}} \mathbf{a}_{k}^{H} \mathbf{n}_{t}\right) \\
& \left(1_{\left(\epsilon_{t, 1}, \epsilon_{t, 2}\right)=(-1,-1)}-(-1)^{k} 1_{\left(\epsilon_{t, 1}, \epsilon_{t, 2}\right)=(-1,+1)}\right. \\
& \left.+(-1)^{k} 1_{\left(\epsilon_{t, 1}, \epsilon_{t, 2}\right)=(+1,-1)}-1_{\left(\epsilon_{t, 1}, \epsilon_{t, 2}\right)=(+1,+1)}\right)
\end{aligned}
$$

${ }^{5}$ For a uniform linear array of $M$ sensors, $g_{1,2}=2 \sigma_{1} \sigma_{2} \cos (\Delta \phi+$ $\left.\frac{(M-1) \Delta \theta}{2}\right)\left(\frac{\sin \left(\frac{M \Delta \theta}{2}\right)}{\sin \left(\frac{\Delta \theta}{2}\right)}\right)$ and $\alpha \approx 0.224$. 
$k=1,2$. Consequently

$$
\begin{aligned}
& \frac{\partial \ln p\left(\mathbf{y}_{t} ; \boldsymbol{\alpha}\right)}{\partial \phi_{1}} \frac{\partial \ln p\left(\mathbf{y}_{t} ; \boldsymbol{\alpha}\right)}{\partial \phi_{2}} \\
& \approx-\frac{\sigma_{1} \sigma_{2}}{\sigma_{n}^{4}}\left(e^{i \phi_{1}} \mathbf{n}_{t}^{H} \mathbf{a}_{1}-e^{-i \phi_{1}} \mathbf{a}_{1}^{H} \mathbf{n}_{t}\right) \\
& \left(e^{i \phi_{2}} \mathbf{n}_{t}^{H} \mathbf{a}_{2}-e^{-i \phi_{2}} \mathbf{a}_{2}^{H} \mathbf{n}_{t}\right) \\
& \left(1_{\left(\epsilon_{t, 1}, \epsilon_{t, 2}\right)=(-1,-1)}-1_{\left(\epsilon_{t, 1}, \epsilon_{t, 2}\right)=(-1,+1)}\right. \\
& \left.-1_{\left(\epsilon_{t, 1}, \epsilon_{t, 2}\right)=(+1,-1)}+1_{\left(\epsilon_{t, 1}, \epsilon_{t, 2}\right)=(+1,+1)}\right) \\
& \frac{\partial \ln p\left(\mathbf{y}_{t} ; \boldsymbol{\alpha}\right)}{\partial \phi_{k}} \frac{\partial \ln p\left(\mathbf{y}_{t} ; \boldsymbol{\alpha}\right)}{\partial \phi_{k}} \\
& \approx-\frac{\sigma_{k}^{2}}{\sigma_{n}^{4}}\left(e^{i \phi_{k}} \mathbf{n}_{t}^{H} \mathbf{a}_{k}-e^{-i \phi_{k}} \mathbf{a}_{k}^{H} \mathbf{n}_{t}\right)^{2} \\
& \left(1_{\left(\epsilon_{t, 1}, \epsilon_{t, 2}\right)=(-1,-1)}+1_{\left(\epsilon_{t, 1}, \epsilon_{t, 2}\right)=(-1,+1)}\right. \\
& \left.+1_{\left(\epsilon_{t, 1}, \epsilon_{t, 2}\right)=(+1,-1)}+1_{\left(\epsilon_{t, 1}, \epsilon_{t, 2}\right)=(+1,+1)}\right)
\end{aligned}
$$

and because $\left(\epsilon_{t, 1}, \epsilon_{t, 2}\right)$ and $\mathbf{n}_{t}$ are independent and the four couples of symbols are equiprobable

$$
\begin{aligned}
\mathrm{E}\left(\frac{\partial \ln p\left(\mathbf{y}_{t} ; \boldsymbol{\alpha}\right)}{\partial \phi_{1}} \frac{\partial \ln p\left(\mathbf{y}_{t} ; \boldsymbol{\alpha}\right)}{\partial \phi_{2}}\right) & \\
\approx-\frac{\sigma_{1} \sigma_{2}}{\sigma_{n}^{4}} \mathrm{E}\left(\left(e^{i \phi_{1}} \mathbf{n}_{t}^{H} \mathbf{a}_{1}-e^{-i \phi_{1}} \mathbf{a}_{1}^{H} \mathbf{n}_{t}\right)\right. & \left.\left(e^{i \phi_{2}} \mathbf{n}_{t}^{H} \mathbf{a}_{2}-e^{-i \phi_{2}} \mathbf{a}_{2}^{H} \mathbf{n}_{t}\right)\right) \\
\left(P \left[\left(\epsilon_{t, 1}, \epsilon_{t, 2}\right)=\right.\right. & (-1,-1)]-P\left[\left(\epsilon_{t, 1}, \epsilon_{t, 2}\right)=(-1,+1)\right] \\
-P\left[\left(\epsilon_{t, 1}, \epsilon_{t, 2}\right)=\right. & \left.(+1,-1)]+P\left[\left(\epsilon_{t, 1}, \epsilon_{t, 2}\right)=(+1,+1)\right]\right) \\
& =0, \\
\mathrm{E}\left(\frac{\partial \ln p\left(\mathbf{y}_{t} ; \boldsymbol{\alpha}\right)}{\partial \phi_{k}} \frac{\partial \ln p\left(\mathbf{y}_{t} ; \boldsymbol{\alpha}\right)}{\partial \phi_{k}}\right) & \approx-\frac{\sigma_{k}^{2}}{\sigma_{n}^{4}} \mathrm{E}\left(\left(e^{i \phi_{k}} \mathbf{n}_{t}^{H} \mathbf{a}_{k}-e^{-i \phi_{k}} \mathbf{a}_{k}^{H} \mathbf{n}_{t}\right)^{2}\right) \\
\left(P \left[\left(\epsilon_{t, 1}, \epsilon_{t, 2}\right)=\right.\right. & (-1,-1)]+P\left[\left(\epsilon_{t, 1}, \epsilon_{t, 2}\right)=(-1,+1)\right] \\
+P\left[\left(\epsilon_{t, 1}, \epsilon_{t, 2}\right)=\right. & \left.(+1,-1)]+P\left[\left(\epsilon_{t, 1}, \epsilon_{t, 2}\right)=(+1,+1)\right]\right) \\
& =\frac{2 M \sigma_{k}^{2}}{\sigma_{n}^{2}} .
\end{aligned}
$$

Using a mixture of 16 Gaussian PDFs, the extension to two QPSK sources follows along the same lines.

\section{REFERENCES}

[1] P. Stoica, A.G. Larsson and A.B. Gershman, "The stochastic CRB for array processing: a textbook derivation," IEEE Signal Processing letters, vol. 8, no. 5, pp. 148-150, May 2001.

[2] "Harmonic retrieval in non-circular complex-valued multiplicative noise: Cramer-Rao bound," Proc. ICASSP Montreal, vol. II, pp. 489-492, May 2004.

[3] M. Haardt, F. Römer, "Enhancements of unitary esprit for non-circular sources," Proc. ICASSP Montreal, vol. II, pp. 101-104, May 2004.

[4] P. Stoica, A. Nehorai, "Performance study of conditional and unconditional direction of arrival estimation," IEEE Trans. on Acoustics Speech and Signal Processing, vol. 38, no. 10, pp. 1783-1795, Oct. 1990.

[5] P. Stoica, R. Moses, Introduction to spectral analysis, PrenticeHall, Upper Saddle River, NJ, 1997.

[6] J.P. Delmas, H. Abeida, "Stochastic Cramer-Rao bound for non-circular signals with application to DOA estimation," IEEE Trans. on Signal Processing, vol. 52, no. 11, pp. 31923199, Nov. 2004.
[7] F. Rice, B. Cowley, B. Moran and M. Rice, "Cramer-Rao lower bounds for QMA phase and frequency estimation," IEEE Trans. on Communications, vol. 49, no. 9, pp. 1582-1591, Sep. 2001.

[8] G.N. Tavales, L.M. Tavares and M.S. Piedade, "Improved Cramer-Rao lower bounds for phase and frequency estimation with $M$-PSK signals," IEEE Trans. on Communications, vol. 49, no. 12 , pp. 2083-2087, Dec. 2001.

[9] B.M. Sadler, R.J. Kozick and T. Moore, "Bounds on bearing and symbol estimation with side information," IEEE Trans. on Signal Processing, vol. 29, no. 4, pp. 822-834, April 2001.

[10] N. S. Alagha, "Cramer-Rao bounds for SNR estimates for BPSK and QPSK modulated signals," IEEE Communications letters, vol. 5, no. 1, pp. 10-12, January 2001.

[11] A. Leshem, A.J. van der Veen, "Direction-of-arrival estimation for constant modulus signals," IEEE Trans. on Communications, vol. 47, no. 11, pp. 3125 -3129, Nov. 1999.

[12] P. Stoica, A. Jakobsson and J. Li, "Cissoid parameter estimation in the colored noise case: asymptotic Cramer-Rao bound, maximum likelihood, and nonlinear least-squares," IEEE, Trans. Signal Processing, vol. 45, no. 8, pp. 2048-2059, August 1997.

[13] J.P. Delmas, "Asymptotically minimum variance secondorder estimation for non-circular signals with application to DOA estimation," IEEE Trans. Signal Processing, vol. 52, no. 5, pp. 1235-1241, May 2004.

[14] B. Ottersten, M. Viberg and T. Kailath, "Analysis of subspace fitting and ML techniques for parameter estimation from sensor array data," IEEE Trans. Signal Processing, vol. 40, no. 3, pp. 590-600, March 1992.

[15] M. Lavielle, E. Moulines and J.F. Cardoso, "A maximum likelihood solution to DOA estimation for discrete sources," Proc. Seventh IEEE Workshop on SP, pp. 349-353, 1994.

[16] P. Chargé, Y. Wang and J. Saillard, "A noncircular sources direction finding method using polynomial rooting," Signal Processing, vol. 81, pp. 1765-1770, 2001.

[17] B. Porat, Digital processing of random signals, Theory and methods, Prentice Hall, 1994.

Jean Pierre Delmas (M'00) was born in France in 1950. He received the Engineering degree from Ecole Centrale de Lyon, France in 1973, the Certificat d'études supérieures from the Ecole Nationale Supérieure des Télécommunications, Paris, France in 1982 and the Habilitation à diriger des recherches (HDR) degree from the University of Paris, Orsay, France in 2001. Since 1980, he has been with the Institut National des Télécommunications where he is presently a Professor in the CITI department and in UMR-CNRS 5157. His teaching and research interests are in the areas of statistical signal processing with application to communications and antenna array. He is currently an Associate Editor for the IEEE Transactions on Signal Processing.

Habti Abeida was born in Settat, Morocco, on october 20, 1977 . He received the Mastery engineering degrees in applied mathematics from Hassan II university, Casablanca, Morocco in 2000 and from René Descartes University, Paris, France in 2001 respectively, and Master's degree in Statistics from Pierre et Marie Curie university, Paris, France, in 2002. He is currently pursuing the $\mathrm{Ph}$. D. degree in applied mathematics and digital communications in the Institut National des Télécommunications, Evry, France. His research interests are in statistical signal processing. 